\title{
Site symmetry and crystal symmetry: a spherical tensor analysis
}

\author{
Christian Brouder, Amélie Juhin, Amélie Bordage, \\ Marie-Anne Arrio \\ Institut de Minéralogie et de Physique des Milieux Condensés, CNRS UMR 7590, \\ Universités Paris 6 et 7, IPGP, 140 rue de Lourmel, 75015 Paris, France. \\ E-mail: christian.brouder@impmc.jussieu.fr

\begin{abstract}
The relation between the properties of a specific crystallographic site and the properties of the full crystal is discussed by using spherical tensors. The concept of spherical tensors is introduced and the way it transforms under the symmetry operations of the site and from site to site is described in detail. The law of spherical tensor coupling is given and illustrated with the example of the electric dipole and quadrupole transitions in x-ray absorption spectroscopy. The main application of the formalism is the reduction of computation time in the calculation of the properties of crystals by band structure methods. The general approach is illustrated by the examples of substitutional chromium in spinel and substitutional vanadium in garnet.
\end{abstract}

Uncomment for PACS numbers title message

PACS numbers: 61.72.S-,61.05.cj,02.20.-a,61.50.Ah 


\section{Introduction}

This paper deals with the relation between the property of specific sites in a crystal and the property of the crystal itself. We shall discuss in particular the case of x-ray absorption spectroscopy, but many of our results are general.

The aim of this paper is to introduce x-ray physicists, experimentalists and theoreticians, to the use of spherical tensors to analyze theoretical and experimental spectra. Therefore, our presentation is as pedagogical as we can manage. The manipulation of spherical tensors can be quite complicated, we have tried to reduce it to the simplest possible rules.

An atom in a crystal occupies generally a crystallographic site that is not unique. The present paper was inspired by the concrete case of a chromium atom sustituted for aluminum in spinel [1]. The chromium atom can occupy sixteen equivalent trigonal sites in the cubic cell. The (normalized) x-ray absorption spectrum of chromium is the average of the spectra of the chromium atoms occupying the sixteen sites. The angular-dependent spectrum of a chromium atom in a specific site is determined by its environment, but some features of this spectrum are lost by the average over the equivalent sites. If we want to investigate the deformation of the environment due to the substitution of chromium for aluminium, we need to know the spectrum of chromium at a given site. But some features of this spectrum are washed out by the fact that we can only measure an average spectrum. The present paper describes precisely the relation between the spectrum of a given site and the spectrum of the crystal.

If we want now to calculate the spectrum of chromium, we need to put a chrominum atom at a specific aluminum site, relax the environment, carry out a self-consistent calculation with and without core hole, and calculate the spectrum of this site. Then, we need to repeat the procedure for all equivalent sites and take the average spectrum. A considerable amount of time can be saved if we calculate the spectrum of one site and deduce the spectrum of the other sites by symmetry considerations. The present paper gives all the tools required to do so.

Let us first defend the case of spherical tensors, that will be precisely defined in the next section. Most physical properties are tensors and they are usually taken to be Cartesian tensors. In this paper, we want to stress the advantage of using spherical tensors. They are a refinement with respect to Cartesian tensor. For example, a second-rank Cartesian tensor is the sum a zeroth-rank, a first-rank and a second-rank spherical tensors. This refinement enables us to discard irrelevant contributions. For example, the quadrupole transitions in x-ray absorption spectroscopy are represented by a fourth-rank Cartesian tensor with 81 components, whereas it is represented by a zeroth-rank, a second-rank and a fourth-rank spherical tensor with 15 components altogether. Moreover, the spherical average used to represent powder sample is obtained by taking only one spherical tensor component (the zeroth-rank tensor) whereas it is the sum of twelve Cartesian components. Thus, using spherical tensors can save computing time. 
The drawback of the spherical tensor analysis is that some subtelties enter its practical use (the same is true for Cartesian tensors). A purpose of this paper is to give a detailed presentation of these problems and of their solutions.

In practice, one faces the frustrating task of sorting out the various conventions used by the authors and to determine whether the rotation is active or passive, if the row index of the Wigner matrix increases from left to right as in a normal matrix or decrease as in reference [2], p. 47, which definition of the Wigner matrix is used (seven inequivalent definitions are found in the literature [3]), how the tensor transforms under complex conjugation, etc. Misprints are also numerous in the literature and we made thorough efforts to give correct formulas.

The paper starts with a presentation of the Cartesian and spherical tensors and a detailed description of the notation. Then, the concept of tensor coupling is introduced and simple formulas are given to change the coupling order. These methods are applied to the calculation of electric dipole and quadrupole transitions. This completes the generalities on spherical tensors. Then, we consider the case of crystals. We show how the symmetries of a crystal site constrains the spherical tensor components in the reference frame of the site and in the reference frame of the crystal. We also give the precise formula relating these reference frames. As an illustration, we treat the example of the Al site in spinel and garnet. Finally, we determine the spherical tensor describing the full crystal from those of the sites. An appendix gathers the formulas that were used to calculate rotation matrices, Wigner matrices and solid harmonics.

\section{Cartesian and spherical tensors}

A Cartesian tensor is the generalization of a scalar, a vector or a matrix. A vector $\mathbf{r}=$ $(x, y, z)$ is transformed by a rotation $R$ into a vector $\mathbf{r}^{\prime}=R \mathbf{r}$, so that $\mathbf{r}_{i}^{\prime}=\sum_{j=1}^{3} R_{i j} \mathbf{r}_{j}$. A vector is also called a first-rank tensor. A matrix $A$ defined by $A_{i j}=\mathbf{r}_{i} \mathbf{r}_{j}$ transforms under rotation into

$$
A_{i j}^{\prime}=\mathbf{r}_{i}^{\prime} \mathbf{r}_{j}^{\prime}=\sum_{l m} R_{i l} R_{j m} A_{l m}
$$

Any matrix $A_{i j}$ that transforms under rotation as in equation (1) is called a second-rank Cartesian tensor. More generally, an $n$ th-rank Cartesian tensor is a generalized matrix $A_{i_{1} \ldots i_{n}}$ that transforms under rotation as in equation (11), but with the product of $n$ matrices $R$ instead of just two. Cartesian tensors are ubiquitous in physics. As we shall see, the electric dipole transitions are described by a second-rank tensor, the quadrupole ones by a fourth-rank tensor.

Cartesian tensors have very simple transformation rules under rotation, but they suffer from a severe drawback: they are not irreducible. To see what that means, consider a second-rank tensor $A_{i j}$. Its trace is $t=\sum_{i=1}^{3} A_{i i}$. It transforms under rotation into

$$
t^{\prime}=\sum_{i=1}^{3} A_{i i}^{\prime}=\sum_{i l m} R_{i l} R_{i m} A_{l m}=\sum_{l m} \delta_{l m} A_{l m}=t
$$


where we used the fact that $R R^{T}=\mathrm{Id}$, where $R^{T}$ is the transpose of $R$. We recover the fact that the trace of a matrix is invariant: it transforms into itself by rotation. A second-order Cartesian tensor gives another interesting object, the vector $\mathbf{v}$, which is defined by $\mathbf{v}_{i}=\sum_{j k} \epsilon_{i j k} A_{j k}$, where the sum is over $j$ and $k$ from 1 to 3 . The LeviCivita symbol $\epsilon_{i j k}$ is 1 if $(i, j, k)$ is a cyclic permutation of $(1,2,3)$, it is -1 if $(i, j, k)$ is another permutation of $(1,2,3)$ and it is 0 if two indices are identical. Then, using the identity $\sum_{j k} \epsilon_{i j k} R_{j l} R_{k m}=\sum_{j} R_{i j} \epsilon_{j l m}$, we see that $\mathbf{v}$ transforms under rotation as a vector: $\mathbf{v}^{\prime}=R \mathbf{v}$. Therefore, from a second-rank Cartesian tensor, we can build a linear combination of its elements (the trace) that is invariant under rotation, and three linear combinations of its elements that transform into each other as the components of a vector. More generally, a tensor is said to be reducible when there are linear combinations of its elements that transform into each other under rotation. When a tensor is not reducible, it is called irreducible. Thus, a vector is irreducible but a secondrank Cartesian tensor is reducible. The irreducible tensors are called spherical tensors and will be the main topic of this paper.

The first spherical tensors were the spherical harmonics $Y_{\ell}^{m}$. For each $\ell$, there

are $2 \ell+1$ spherical harmonics $Y_{\ell}^{m}$ that transform into each other by rotation. More precisely, for each rotation $R$, there is a unitary matrix $D^{\ell}$, called a Wigner matrix (to be precisely defined in the next section), such that the rotation of $Y_{\ell}^{m}$ by $R$ is

$$
R Y_{\ell}^{m}=\sum_{m^{\prime}=-\ell}^{\ell} Y_{\ell}^{m^{\prime}} D_{m^{\prime} m}^{\ell}(R) .
$$

Spherical tensors are defined in analogy with spherical harmonics. An $\ell$ th rank spherical tensor, denoted by $T^{\ell}$, is a set of $2 \ell+1$ components, written $T_{m}^{\ell}$ where $m=-\ell,-\ell+1, \ldots, \ell-1, \ell$, that transform under rotation as

$$
R T_{m}^{\ell}=\sum_{m^{\prime}=-\ell}^{\ell} T_{m^{\prime}}^{\ell} D_{m^{\prime} m}^{\ell}(R) .
$$

This definition is not very concrete, but we shall see how spherical tensors are built in practice. It is an unfortunate but historical fact that the position of $\ell$ and $m$ is different in the spherical harmonics $Y_{\ell}^{m}$ and the spherical tensors $T_{m}^{\ell}$.

When many spherical tensors are involved in a formula, we use also the notation $P^{a}$, $Q^{b}$, etc. For notational convenience, we shall often write $R T^{\ell}=T^{\ell} D^{\ell}(R)$ for equation (2), as for the product of a matrix and a vector. Moreover, the product of two Wigner matrices will be written $D^{\ell}(R) D^{\ell}\left(R^{\prime}\right)$.

\subsection{Further symmetries}

Spherical tensors can satisfy other symmetries. For example, most of them are built from Hermitian operators and satisfy time-reversal symmetry: (see equation (4) p. 61 of [3]).

$$
\left(T_{m}^{\ell}\right)^{*}=(-1)^{m} T_{-m}^{\ell} .
$$


In this paper, the only tensors that do not satisfy time-reversal symmetry are those built from the polarization vector $\boldsymbol{\epsilon}$ that can possibly be complex. In that case we have $\left(T_{m}^{\ell}(\boldsymbol{\epsilon})\right)^{*}=(-1)^{m} T_{-m}^{\ell}\left(\boldsymbol{\epsilon}^{*}\right)$. This happens for instance when $T^{\ell}$ is a solid harmonics (see section 8.2).

Inversion is another common symmetry. In pure electric dipole and quadrupole transitions inversion does not play any role because all tensors are even (i.e. invariant under inversion). However, the interference between dipole and quadrupole transitions is always an odd spherical tensor [4, 5, 6].

\section{Notation}

The many conventions and misprints found in the literature forces us to describe in detail our notation and conventions.

We consider active rotation, that is rotation that move the points and not the reference frame. For example the rotation through an angle $\psi$ about the $z$-axis is represented by

$$
R_{z}(\psi)=\left(\begin{array}{ccc}
\cos \psi & -\sin \psi & 0 \\
\sin \psi & \cos \psi & 0 \\
0 & 0 & 1
\end{array}\right)
$$

After an active rotation $R$, the coordinates $\left(\mathbf{r}_{1}, \mathbf{r}_{2}, \mathbf{r}_{3}\right)$ of the vector $\mathbf{r}$ are transformed into the coordinates $\mathbf{r}_{i}^{\prime}=\sum_{j} R_{i j} \mathbf{r}_{j}$ of $\mathbf{r}^{\prime}=R \mathbf{r}$. In a passive rotation, the reference frame is rotated: the basis vectors $\mathbf{e}_{i}$ are transformed into the basis vectors $\mathbf{e}_{i}^{\prime}=\sum_{j} R_{i j} \mathbf{e}_{j}$. Thus, the coordinates of a point $\mathbf{r}$ are transformed by the inverse matrix: $\mathbf{r}^{\prime}=R^{-1} \mathbf{r}$.

To describe the transformation of the properties of a crystal under rotation, we consider the case of its charge density $\rho(\mathbf{r})$. After a rotation changing $\mathbf{r}$ into $\mathbf{r}^{\prime}=R \mathbf{r}$, the charge density $\rho$ is transformed into a "rotated" charge density $\rho^{\prime}$ of the rotated crystal. To determine $\rho^{\prime}$, we require that the value of the charge density is not modified by the rotation. More precisely, we want that $\rho^{\prime}\left(\mathbf{r}^{\prime}\right)=\rho(\mathbf{r})$. Therefore, the rotated function $\rho^{\prime}$ is defined by $\rho^{\prime}\left(\mathbf{r}^{\prime}\right)=\rho\left(R^{-1} \mathbf{r}^{\prime}\right)$. For later convenience, we denote the rotated function $\rho^{\prime}$ by $R \rho$. The use of the same symbol $R$ to denote the rotation of both the vectors and the functions should not bring too much confusion. The presence of the inverse rotation $R^{-1}$ in the definition of $R \rho$ ensures that $R^{\prime}(R \rho)=\left(R^{\prime} R\right) \rho$ (see Ref. [7] p. 59).

\subsection{Wigner rotation matrices}

We denote by $D_{m^{\prime} m}^{\ell}(R)$ the Wigner rotation matrix corresponding to the rotation $R$. For example, $D_{m^{\prime} m}^{\ell}\left(R_{z}(\psi)\right)=\delta_{m m^{\prime}} \mathrm{e}^{-i m \psi}$. The Wigner rotation matrices define a unitary representation of the rotation group, so that

$$
D_{m^{\prime} m}^{\ell}\left(R^{-1}\right)=\left(D_{m m^{\prime}}^{\ell}(R)\right)^{*}
$$


and

$$
D_{m^{\prime} m}^{\ell}\left(R R^{\prime}\right)=\sum_{m^{\prime \prime}=-\ell}^{\ell} D_{m^{\prime} m^{\prime \prime}}^{\ell}(R) D_{m^{\prime \prime} m}^{\ell}\left(R^{\prime}\right)
$$

\subsection{Spherical harmonics}

The spherical harmonics are defined by (see Ref. [2] p. 68)

$$
Y_{\ell}^{m}(\theta, \phi)=\sqrt{\frac{2 \ell+1}{4 \pi}}\left(D_{m 0}^{\ell}\left(R_{\theta \phi}\right)\right)^{*},
$$

where $R_{\theta \phi}$ is the rotation defined by the Euler angles $(\phi, \theta, 0)$. For notational convenience, we denote by $\mathbf{n}$ the vector $(\sin \theta \cos \phi, \sin \theta \sin \phi, \cos \theta)$ and we write $Y_{\ell}^{m}(\mathbf{n})$ and $R_{\mathbf{n}}$ for $Y_{\ell}^{m}(\theta, \phi)$ and $R_{\theta \phi}$. This notation is justified by the fact that $Y_{\ell}^{m}(\mathbf{n})$ can be defined for any vector $\mathbf{r}$, not necessarily normalized. The resulting functions are called solid harmonics and are described in section [8.2. Solid harmonics are required, for example, in the presence of elliptically polarized x-rays because $\mathbf{n}$ has then complex coordinates. A three-dimensional Cartesian vector $\mathbf{r}=(x, y, z)$ can be turned into a set of three solid harmonics

$$
\begin{aligned}
Y_{1}^{-1}(\mathbf{r}) & =\sqrt{\frac{3}{8 \pi}}(x-i y), \\
Y_{1}^{0}(\mathbf{r}) & =\sqrt{\frac{3}{4 \pi}} z, \\
Y_{1}^{1}(\mathbf{r}) & =-\sqrt{\frac{3}{8 \pi}}(x+i y) .
\end{aligned}
$$

This definition of spherical harmonics implies

$$
Y_{\ell}^{m}(R \mathbf{n})=\sum_{m^{\prime}} Y_{\ell}^{m^{\prime}}(\mathbf{n}) D_{m^{\prime} m}^{\ell}\left(R^{-1}\right)
$$

This is proved by noticing that the argument $R \mathbf{n}$ of the spherical harmonics corresponds to the argument $R R_{\mathbf{n}}$ of the Wigner matrix in equation (6). By equation (4), we have $\left(D_{m 0}^{\ell}\left(R R_{\mathbf{n}}\right)\right)^{*}=D_{0 m}^{\ell}\left(\left(R R_{\mathbf{n}}\right)^{-1}\right)$. The result follows from $\left(R R_{\mathbf{n}}\right)^{-1}=R_{\mathbf{n}}^{-1} R^{-1}$ and the group representation property, equation (5). The same property is true for solid harmonics. Therefore,

$$
\left(R Y_{\ell}^{m}\right)(\mathbf{r})=Y_{\ell}^{m}\left(R^{-1} \mathbf{r}\right)=\sum_{m^{\prime}} Y_{\ell}^{m^{\prime}}(\mathbf{r}) D_{m^{\prime} m}^{\ell}(R)
$$

The presence of the spherical harmonics on the left of the Wigner rotation matrices ensures that $R^{\prime}\left(R Y_{\ell}^{m}\right)=\left(R^{\prime} R\right) Y_{\ell}^{m}$. To show this, multiply equation (10) on the left by $R^{\prime}$ :

$$
\begin{aligned}
\left(R^{\prime}\left(R Y_{\ell}\right)\right)(\mathbf{r}) & =\left(R^{\prime} Y_{\ell}\right)(\mathbf{r}) D^{\ell}(R)=Y_{\ell}(\mathbf{r}) D^{\ell}\left(R^{\prime}\right) D^{\ell}(R) \\
& =Y_{\ell}(\mathbf{r}) D^{\ell}\left(R^{\prime} R\right)=\left(R^{\prime} R\right) Y_{\ell}(\mathbf{r})
\end{aligned}
$$

In the foregoing proof, we simplified the notation by omitting the component index $m$ (as described at the end of section 2). 


\section{Building tensor operators}

Physical properties can be represented by spherical tensors that can often be built by coupling lower rank tensors. We illustrate this construction by the example of electric dipole and quadrupole transitions. We shall use the remarkable toolbox for spherical tensor calculations elaborated by Varshalovich, Moskalev and Khersonskii [3].

Many spherical tensors used in physics are obtained by coupling vectors. A threedimensional Cartesian vector $\mathbf{v}=(x, y, z)$ can be turned into a first-rank spherical tensor $\mathbf{v}^{1}$ by defining

$$
\begin{aligned}
& \mathbf{v}_{-1}^{1}=(x-i y) / \sqrt{2} \\
& \mathbf{v}_{0}^{1}=z, \\
& \mathbf{v}_{1}^{1}=-(x+i y) / \sqrt{2} .
\end{aligned}
$$

Note that solid harmonics $Y_{\ell}^{m}(\mathbf{v})$ are also spherical tensors built from $\mathbf{v}$ and that $\mathbf{v}^{1}=Y_{1}(\mathbf{v}) \sqrt{4 \pi / 3}$. However, the factor $\sqrt{4 \pi / 3}$ is cumbersome and the definition $\mathbf{v}^{\ell}=Y_{\ell}(\mathbf{v}) \sqrt{4 \pi /(2 \ell+1)}$ is often preferred.

Two spherical tensors $P^{a}$ and $Q^{b}$ of ranks $a$ and $b$ can be coupled into a spherical tensor of rank $c$, denoted by $\left\{P^{a} \otimes Q^{b}\right\}^{c}$, and defined by

$$
\left\{P^{a} \otimes Q^{b}\right\}_{\gamma}^{c}=\sum_{\alpha=-a}^{a} \sum_{\beta=-b}^{b}(a \alpha b \beta \mid c \gamma) P_{\alpha}^{a} Q_{\beta}^{b},
$$

where $(a \alpha b \beta \mid c \gamma)$ are Clebsch-Gordan coefficients [2, 3]. The Clebsch-Gordan coefficient is zero if $\gamma \neq \alpha+\beta$. The triangle relation implies $|a-b| \leq c \leq a+b$. The notation $\left\{P^{a} \otimes Q^{b}\right\}^{c}$ is inspired by ref. [3], except for the fact that they write the rank as an index instead of an exponent. For example, the coupling of two vectors (i.e. first-rank spherical tensors) gives a zeroth-rank, a first-rank and a second-rank spherical tensors. The zeroth-rank tensor obtained by coupling two vectors is proportional to the scalar product of these vectors: $\left\{\mathbf{u}^{1} \otimes \mathbf{v}^{1}\right\}^{0}=-\mathbf{u} \cdot \mathbf{v} / \sqrt{3}$, because $(1 \alpha 1-\alpha \mid 00)=-(-1)^{\alpha} / \sqrt{3}$. More generally, we define the scalar product of two spherical tensors $P^{a}$ and $Q^{a}$ of the same rank to be (see [3], p. 64 and 65)

$$
P^{a} \cdot Q^{a}=\sum_{\alpha=-a}^{a}(-1)^{\alpha} P_{-\alpha}^{a} Q_{\alpha}^{a}=(-1)^{a} \sqrt{2 a+1}\left\{P^{a} \otimes Q^{a}\right\}^{0} .
$$

It is often necessary to modify the coupling order of the tensors. For example, when calculating electric dipole transitions, we have to calculate $|\langle f|\boldsymbol{\epsilon} \cdot \mathbf{r}| i\rangle|^{2}$, where $\mathbf{r}$ is coupled to $\boldsymbol{\epsilon}$ by the scalar product and then multiplied by its complex conjugate. As we shall see in the next section, it is more convenient from the physical point of view to couple the x-ray polarization vectors $\boldsymbol{\epsilon}$ and $\boldsymbol{\epsilon}^{*}$. For that purpose, there is a powerful identity

$$
\left\{P^{a} \otimes Q^{a}\right\}^{0} \cdot\left\{R^{d} \otimes S^{d}\right\}^{0}=\sum_{g}(-1)^{g} \frac{\left\{P^{a} \otimes R^{d}\right\}^{g} \cdot\left\{Q^{a} \otimes S^{d}\right\}^{g}}{\sqrt{(2 a+1)(2 d+1)}}
$$


where $g$ runs from $|a-d|$ to $a+d$ by the triangle relation. This identity is proved in section 9 .

In the next two sections, we illustrate the recoupling methods with the calculation of electric dipole and quadrupole transitions. Similar methods were used to investigate the interference of electric and quadrupole transitions and their interferences [5, 6, 8, or to calculate x-ray scattering cross-sections [9].

\subsection{Dipole}

The electric dipole transitions amplitudes are given by the formula $T_{f i}=\langle f|\boldsymbol{\epsilon} \cdot \mathbf{r}| i\rangle$. If we denote $\langle f|\mathbf{r}| i\rangle$ by $\mathbf{r}_{f i}$, equation (12) gives us $T_{f i}=\boldsymbol{\epsilon} \cdot \mathbf{r}_{f i}=-\sqrt{3}\left\{\boldsymbol{\epsilon}^{1} \otimes \mathbf{r}_{f i}^{1}\right\}^{0}$. For notational convenience, we remove the exponent 1 in the spherical tensors $\boldsymbol{\epsilon}^{1}$ and $\mathbf{r}_{f i}^{1}$. This should not bring confusion: if a vector takes part in a coupling, it is a first-rank tensor. Using the recoupling identity (13), we find the dipole transition intensity

$$
\left|T_{f i}\right|^{2}=3\left\{\boldsymbol{\epsilon}^{*} \otimes \mathbf{r}_{f i}^{*}\right\}^{0}\left\{\boldsymbol{\epsilon} \otimes \mathbf{r}_{f i}\right\}^{0}=\sum_{a=0}^{2}(-1)^{a}\left\{\boldsymbol{\epsilon}^{*} \otimes \boldsymbol{\epsilon}\right\}^{a} \cdot\left\{\mathbf{r}_{f i}^{*} \otimes \mathbf{r}_{f i}\right\}^{a} .
$$

Note that, for elliptic polarization, $\boldsymbol{\epsilon}$ is complex, whereas the wavevector $\mathbf{k}$ is always real. Each term of a decomposition over spherical tensors has often a clear physical meaning. In equation (14), the variables concerning the incident x-ray (i.e. $\boldsymbol{\epsilon}$ and $\boldsymbol{\epsilon}^{*}$ ) are gathered in $\left\{\boldsymbol{\epsilon}^{*} \otimes \boldsymbol{\epsilon}\right\}^{a}$, the variables concerning the crystal are in $\left\{\mathbf{r}_{f i}^{*} \mathbf{r}_{f i}\right\}^{a}$. Thus, we can easily investigate the influence of a rotation $R$ of the crystal on the absorption cross-section

$$
\begin{aligned}
R\left|T_{f i}\right|^{2} & =\sum_{a=0}^{2}(-1)^{a}\left\{\boldsymbol{\epsilon}^{*} \otimes \boldsymbol{\epsilon}\right\}^{a} \cdot\left(R\left\{\mathbf{r}_{f i}^{*} \otimes \mathbf{r}_{f i}\right\}^{a}\right) \\
& =\sum_{a=0}^{2}(-1)^{a}\left\{\boldsymbol{\epsilon}^{*} \otimes \boldsymbol{\epsilon}\right\}^{a} \cdot\left(\left\{\mathbf{r}_{f i}^{*} \otimes \mathbf{r}_{f i}\right\}^{a} D^{a}(R)\right),
\end{aligned}
$$

where we used equation (2) and that $\left\{\mathbf{r}_{f i}^{*} \otimes \mathbf{r}_{f i}\right\}^{a}$ is a ath-rank spherical tensor. In particular, the spectrum of a powder sample is given by the average over all orientations, i.e. over all rotations $R$. This average is very simple with spherical tensors $\left\langle D^{a}(R)\right\rangle=$ $\delta_{a, 0}$. Thus, the term $a=0$ gives the spectrum of a powder, called the isotropic spectrum.

$$
\left\langle\left|T_{f i}\right|^{2}\right\rangle=\left\{\boldsymbol{\epsilon}^{*} \otimes \boldsymbol{\epsilon}\right\}^{0} \cdot\left\{\mathbf{r}_{f i}^{*} \otimes \mathbf{r}_{f i}\right\}^{0}=\frac{1}{3}\left(\boldsymbol{\epsilon}^{*} \cdot \boldsymbol{\epsilon}\right)\left(\mathbf{r}_{f i}^{*} \cdot \mathbf{r}_{f i}\right)=\frac{\left|\mathbf{r}_{f i}\right|^{2}}{3},
$$

where we used equation (12) and $|\boldsymbol{\epsilon}|^{2}=1$.

To interpret the term $a=1$, we use the relation between vectors $\mathbf{u}, \mathbf{v}$ and the corresponding first-rank tensors $\mathbf{u}^{1}, \mathbf{v}^{1}$ (we restore the tensor rank in $\mathbf{u}^{1}, \mathbf{v}^{1}$ for clarity): according to equation (27), $\left\{\mathbf{u}^{1} \otimes \mathbf{v}^{1}\right\}^{1}$ is the first-rank tensor corresponding to the vector $(i / \sqrt{2}) \mathbf{u} \times \mathbf{v}$. Therefore,

$$
\left\{\boldsymbol{\epsilon}_{\otimes}^{*} \boldsymbol{\epsilon}\right\}^{1} \cdot\left\{\mathbf{r}_{f i}^{*} \otimes \mathbf{r}_{f i}\right\}^{1}=-\frac{1}{2}\left(\boldsymbol{\epsilon}^{*} \times \boldsymbol{\epsilon}\right) \cdot\left(\mathbf{r}_{f i}^{*} \times \mathbf{r}_{f i}\right) .
$$

The first cross product is related to the rate of circular polarization $P_{c}$ and to wavevector direction $\hat{k}$ of the incident $\mathrm{x}$-ray by $\boldsymbol{\epsilon}^{*} \times \boldsymbol{\epsilon}=-i P_{c} \hat{k}[5]$. Moreover, the second cross 
product is zero for a non-magnetic sample because time-reversal symmetry implies $\mathbf{r}_{f i}^{*}=\mathbf{r}_{f i}$. Therefore, the term $a=1$ describes magnetic circular dichroism.

The term $a=2$ describes the linear dichroism of x-ray spectra. The number of non-zero components of $\left\{\mathbf{r}_{f i}^{*} \otimes \mathbf{r}_{f i}\right\}^{2}$ depends on the symmetry of the crystal [10]. We shall determine the number of non-zero components in the case of spinel and garnet.

\subsection{Quadrupole}

We consider the case of electric quadrupole transitions, which is more involved than dipole transitions. We start from the quadrupole transition operator $T=\boldsymbol{\epsilon} \cdot \mathbf{r} \mathbf{k} \cdot \mathbf{r}$ and we rewrite it in terms of spherical tensors using equation (28): $T=3\left\{\{\boldsymbol{\epsilon} \otimes \mathbf{r}\}^{0} \otimes\{\mathbf{k} \otimes \mathbf{r}\}^{0}\right\}^{0}$. In this expression $\boldsymbol{\epsilon}$ is coupled with $\mathbf{r}$ and $\mathbf{k}$ with $\mathbf{r}$. As in the electric dipole, we want to gather all the terms concerning the crystal into a single tensor. For that purpose, we use equation (13) with the sum over $g$ changed into a sum over $a$

$$
T=\sum_{a=0}^{2}(-1)^{a}\{\boldsymbol{\epsilon} \otimes \mathbf{k}\}^{a} \cdot\{\mathbf{r} \otimes \mathbf{r}\}^{a} .
$$

The term $a=0$ is zero because, according to equation (12), $\{\boldsymbol{\epsilon} \otimes \mathbf{k}\}^{0}=-(1 / \sqrt{3}) \boldsymbol{\epsilon} \cdot \mathbf{k}=0$ since the polarization and wavevectors are perpendicular. The term $a=1$ is zero because equation (27) gives us $\{\mathbf{r} \otimes \mathbf{r}\}^{1}=(i / \sqrt{2}) \mathbf{r} \times \mathbf{r}=0$. Thus, $T$ is reduced to the single term

$$
T=\{\boldsymbol{\epsilon} \otimes \mathbf{k}\}^{2} \cdot\{\mathbf{r} \otimes \mathbf{r}\}^{2}=\sqrt{5}\left\{\{\boldsymbol{\epsilon} \otimes \mathbf{k}\}^{2} \otimes\{\mathbf{r} \otimes \mathbf{r}\}^{2}\right\}^{0} .
$$

The tensor $\{\mathbf{r} \otimes \mathbf{r}\}^{2}$ can be expressed in terms of spherical harmonics (equation (23), p. 67 of [3])

$$
\{\mathbf{r} \otimes \mathbf{r}\}_{m}^{2}=\sqrt{\frac{8 \pi}{15}} Y_{2}^{m}(\mathbf{r})=\sqrt{\frac{8 \pi}{15}} r^{2} Y_{2}^{m}(\theta, \phi),
$$

where $r, \theta$ and $\phi$ are the spherical coordinates of $\mathbf{r}$. For completeness, we give the components of $\{\boldsymbol{\epsilon} \otimes \mathbf{k}\}^{2}$ :

$$
\begin{aligned}
& \{\boldsymbol{\epsilon} \otimes \mathbf{k}\}_{ \pm 2}^{2}=\frac{\left(\epsilon_{x} \pm i \epsilon_{y}\right)\left(k_{x} \pm i k_{y}\right)}{2} \\
& \{\boldsymbol{\epsilon} \otimes \mathbf{k}\}_{ \pm 1}^{2}=\mp \frac{\left(\epsilon_{x} \pm i \epsilon_{y}\right) k_{z}+\epsilon_{z}\left(k_{x} \pm i k_{y}\right)}{2} \\
& \{\boldsymbol{\epsilon} \otimes \mathbf{k}\}_{0}^{2}==\frac{3 \epsilon_{z} k_{z}-\boldsymbol{\epsilon} \cdot \mathbf{k}}{\sqrt{6}}=\sqrt{\frac{3}{2}} \epsilon_{z} k_{z} .
\end{aligned}
$$

The electric quadrupole transition intensities are proportional to $\left|T_{f i}\right|^{2}$, where the transition amplitude is $T_{f i}=\langle f|T| i\rangle$. Therefore, $\left|T_{f i}\right|^{2}=5\left\{P^{2} \otimes Q^{2}\right\}^{0}\left\{R^{2} \otimes S^{2}\right\}^{0}$, with $P^{2}=\left\{\boldsymbol{\epsilon}^{*} \otimes \mathbf{k}\right\}^{2}, Q^{2}=\left\langle f\left|\{\mathbf{r} \otimes \mathbf{r}\}^{2}\right| i\right\rangle^{*}, R^{2}=\{\boldsymbol{\epsilon} \otimes \mathbf{k}\}^{2}, S^{2}=\left\langle f\left|\{\mathbf{r} \otimes \mathbf{r}\}^{2}\right| i\right\rangle$,

$$
\left|T_{f i}\right|^{2}=5\left\{P^{2} \otimes Q^{2}\right\}^{0}\left\{R^{2} \otimes S^{2}\right\}^{0}=\sum_{a=0}^{4}(-1)^{a}\left\{P^{2} \otimes R^{2}\right\}^{a} \cdot\left\{Q^{2} \otimes S^{2}\right\}^{a} .
$$


The apparent simplicity of this calculation is essentially due to the powerful tools given in reference [3]. A straightforward approach is quite heavy [10]. If the sample is nonmagnetic or the x-ray polarization is linear, the terms $a=1$ and $a=3$ are zero.

As in the electric dipole case, the term $a=0$ corresponds to the isotropic spectrum

obtained by measuring a powder. Equation (29) gives us $\left\{P^{2} \otimes R^{2}\right\}^{0}=k^{2} /(2 \sqrt{5})$ and the isotropic spectrum is

$$
\left\langle\left|T_{f i}\right|^{2}\right\rangle=k^{2} \frac{\left\{Q^{2} \otimes S^{2}\right\}^{0}}{2 \sqrt{5}} .
$$

The calculation of this average will be discussed in section 7 ,

If $P^{2}=R^{2}$ (i.e. the x-rays are linearly polarized) or $Q^{2}=S^{2}$ (i.e. the sample is non magnetic), then the terms $a=1$ and $a=3$ are zero. More generally, for any tensor $T^{a}$ with integer rank $a,\left\{T^{a} \otimes T^{a}\right\}^{c}$ is zero for $c$ odd. This is due to the symmetry of the Clebsch-Gordan coefficients [2, 3] $(b \beta a \alpha \mid c \gamma)=(-1)^{a+b-c}(a \alpha b \beta \mid c \gamma)$ :

$$
\begin{aligned}
\left\{T^{a} \otimes T^{a}\right\}_{\gamma}^{c} & =\sum_{\alpha, \beta}(a \alpha a \beta \mid c \gamma) T_{\alpha}^{a} T_{\beta}^{a}=(-1)^{2 a-c} \sum_{\beta, \alpha}(a \alpha a \beta \mid c \gamma) T_{\beta}^{a} T_{\alpha}^{a} \\
& =(-1)^{c}\left\{T^{a} \otimes T^{a}\right\}_{\gamma}^{c},
\end{aligned}
$$

where we first exchanged the summation variables $\alpha$ and $\beta$, and used the symmetry of the Clebsch-Gordan coefficients, then the commutativity of $T_{\alpha}^{a}$ and $T_{\beta}^{a}$, and the fact that $a$ is an integer.

If we consider only the case of linearly polarized x-rays or non-magnetic samples, only the terms $a=0, a=2$ and $a=4$ are possibly non zero. The number of independent components depends on the crystal symmetry and is tabulated in [10].

\section{Site symmetry}

We describe how to calculate the spherical tensor of a crystallographic site, assuming that it is invariant under the symmetries of the site. We can work in a reference frame corresponding either to the site symmetries or to the crystal symmetries. If we take the aluminum sites of spinels as an example, the site symmetry is $D_{3 d}$ whereas the crystal is cubic. In the reference frame adapted to the site symmetry, the $z$ axis is along a diagonal of the cube. In the reference frame adapated to the crystal, the $z$ axis is along an edge of the cube. The symmetrized tensors has usually less nonzero components in the site axes, but they are easier to calculate in the crystal axes. We also give the way to go from one reference frame to the other. Two examples are treated in detail: a spinel and a garnet.

\subsection{Symmetrized tensor}

If $G$ is the symmetry group of the crystal, then a site has a symmetry group $G^{\prime}$ which is a subgroup of $G$. To know the general form of a spherical tensor invariant under the site symmetries, we start from a general tensor $T^{\ell}$ and we calculate the symmetrized 
tensor $\left\langle T_{m}^{\ell}\right\rangle$ compatible with the site symmetries by using the classical formula

$$
\left\langle T_{m}^{\ell}\right\rangle=\frac{1}{\left|G^{\prime}\right|} \sum_{R^{\prime}} \sum_{m^{\prime}=-\ell}^{\ell} T_{m^{\prime}}^{\ell} D_{m^{\prime} m}^{\ell}\left(R^{\prime}\right),
$$

where $R^{\prime}$ runs over the symmetry operations of the subgroup $G^{\prime}$ and where $\left|G^{\prime}\right|$ is the number of elements of $G^{\prime}$. From the physical point of view, this formula means that the tensor $\left\langle T_{m}^{\ell}\right\rangle$ is obtained by averaging over all the symmetry operations that leave the site invariant. From the mathematical point of view, we project onto the subset of tensors that are invariant by any symmetry operation of $G^{\prime}$. To check this, take any operation $R$ in $G^{\prime}$ and evaluate the action of $R$ on the symmetrized tensor:

$$
\begin{aligned}
\left\langle R T_{m}^{\ell}\right\rangle & =\frac{1}{\left|G^{\prime}\right|} \sum_{R^{\prime}} \sum_{m^{\prime} m^{\prime \prime}} T_{m^{\prime \prime}}^{\ell} D_{m^{\prime \prime} m^{\prime}}^{\ell}(R) D_{m^{\prime} m}^{\ell}\left(R^{\prime}\right) \\
& =\frac{1}{\left|G^{\prime}\right|} \sum_{R^{\prime}} \sum_{m^{\prime \prime}} T_{m^{\prime \prime}}^{\ell} D_{m^{\prime \prime} m}^{\ell}\left(R R^{\prime}\right)=\left\langle T_{m}^{\ell}\right\rangle,
\end{aligned}
$$

because, $G^{\prime}$ being a group, the set of operations $R R^{\prime}$ where $R^{\prime}$ runs over $G^{\prime}$ is the same as the set of operations of $G^{\prime}$.

\subsection{Site and crystal axes}

The rotations $R$ can be expressed either in the site axes or in the crystal axes. We shall see in the examples that the symmetric tensors are simpler in the site axes. Moreover, some computer programs need to be used in the site axes $₫$ However, the matrices $R$ are easier to determine in the crystal axes, because they correspond to the symmetry operations of the crystal that leave the site invariant. Both cases will be treated in the examples of the following sections.

It is also necessary to describe precisely how to go from one reference frame to the other. If $\mathbf{e}_{1}, \mathbf{e}_{2}, \mathbf{e}_{3}$ are the orthonormal axes associated with the crystal and $\mathbf{e}_{1}^{\prime}$, $\mathbf{e}_{2}^{\prime}, \mathbf{e}_{3}^{\prime}$ those of the site, we denote by $R$ the rotation matrix such that $\mathbf{e}_{i}^{\prime}=\sum_{j} R_{i j} \mathbf{e}_{j}$. For example, if $\mathbf{e}_{i}$ are the axes of the cube and $\mathbf{e}_{i}^{\prime}$ are the trigonal axes along the $(-1,1,1)$ direction, then $R$ is the rotation matrix corresponding to the Euler angles $(0, \arccos (1 / \sqrt{3}), \pi / 4)$. It is the inverse of the rotation matrix of equation (26) $)$. It can be checked that $\mathbf{e}_{3}^{\prime}=\left(-\mathbf{e}_{1}+\mathbf{e}_{2}+\mathbf{e}_{3}\right) / 3$ (i.e. the three-fold axis is the $z$-axis of the site along the $(-1,1,1)$ direction of the cube) and $\mathbf{e}_{2}^{\prime}=\left(\mathbf{e}_{1}+\mathbf{e}_{2}\right) / \sqrt{2}$ (i.e. the $y$ axis of the site is along the $(1,1,0)$ direction of the cube). If $R^{\prime}$ is a symmetry operation in the cubic axes of the cube leaving the site invariant, the basis change formula in a vector space implies that $R R^{\prime} R^{-1}$ is a symmetry operation in the trigonal axes of the site. A spherical tensor $T^{\ell}$ will be denoted by $T^{\ell}(3)$ when it is expressed in the trigonal axes and by $T^{\ell}(4)$ when it is expressed in the cubic axes. The argument 3 and 4 mean that the $z$ axis is along a three-fold axis for a trigonal basis and a four-fold axis for a cubic

$\ddagger$ The most prominent example is the series of multiplet programs written by Cowan, Butler, Thole, Ogasawara and Searle 11, 12, 13, 14, 15. 
basis. The relation between $T^{\ell}(3)$ and $T^{\ell}(4)$ is given by the formula

$$
T_{m}^{\ell}(4)=\sum_{m^{\prime}} T_{m^{\prime}}^{\ell}(3) D_{m^{\prime} m}^{\ell}(R)
$$

We give now two examples.

\subsection{The example of spinel}

We illustrate this method with the example of the aluminium site in spinel $\mathrm{MgAl}_{2} \mathrm{O}_{4}$, which is the most common mineral of the spinel structural family. The spinel structure is derived from a face-centred-cubic close-packing of oxygens with a space group symmetry $F d \overline{3} \mathrm{~m}$. The conventional cubic cell contains 8 formula units, i.e. 32 oxygen atoms with 24 cations in tetrahedral and pseudo-octahedral interstices. With origin choice 2 [16], the $\mathrm{Mg}^{2+}$ cations occupy 8 tetrahedral sites, which are located at the special 8a Wyckoff positions $(1 / 8,1 / 8,1 / 8)$, with $\overline{4} 3 m\left(T_{d}\right)$ point symmetry. The $\mathrm{Al}^{3+}$ cations occupy 16 pseudo-octahedral sites at the special 16d Wyckoff positions $(0,1 / 4,3 / 4)$, with $\overline{3} m\left(D_{3 d}\right)$ point symmetry. This symmetry corresponds to a small elongation of the octahedron along the trigonal axis, arising from a small departure of the position of the oxygens from the perfect fcc arrangement. The $\mathrm{O}^{2-}$ ions are located at the Wyckoff positions $32 \mathrm{e}(\mathrm{u}, \mathrm{u}, \mathrm{u})$ with point symmetry $3 \mathrm{~m}$.

5.3.1. The site frame The simplest results are obtained when the reference frame of the site is used. The point group of the site we consider is $D_{3 d}$. The group $D_{3 d}$ has six pure rotations and the same six rotations multiplied by the inversion. We assume that the property that we investigate is not sensitive to the inversion operator, so that we only have to consider the six pure rotations. It is natural to take the $z$-axis along the three-fold axis and the $y$ axis along one of the $C_{2}$ axes. The results do not depend on which $C_{2}$ axis is chosen. However, they would be different if the $y$ axis were chosen, for example, between two $C_{2}$ axes. The six pure rotations are: the unit, the $C_{3}$ rotation about the $z$-axis through the angle $2 \pi / 3$, its square $C_{3}^{2}$, the $C_{2}$ rotation about the $y$ axis through the angle $\pi$ and the other two rotations $C_{3} C_{2}$ and $C_{3}^{2} C_{2}$. These rotations have Euler angles $(0,0,0),(0,0,2 \pi / 3),(0,0,4 \pi / 3),(0, \pi, 0),(0, \pi, 4 \pi / 3)$ and $(0, \pi, 2 \pi / 3)$, respectively. These rotations will be denoted by $R_{1}, \ldots, R_{6}$.

To calculate the symmetrized tensors for this site, we use equation (16). The special cases given in section 8.3 .1 enable us to show that $D_{m^{\prime} m}^{\ell}\left(R_{1}\right)=\delta_{m^{\prime} m}, D_{m^{\prime} m}^{\ell}\left(R_{2}\right)=$ $\delta_{m^{\prime} m} \mathrm{e}^{-2 m i \pi / 3}$ and $D_{m^{\prime} m}^{\ell}\left(R_{3}\right)=\delta_{m^{\prime} m} \mathrm{e}^{-4 m i \pi / 3}$. Therefore, the sum $\sum_{j=1}^{3} D_{m^{\prime} m}^{\ell}\left(R_{j}\right)$ is $3 \delta_{m^{\prime} m}$ if $m$ is an integer multiple of 3 , and zero otherwise. We calculate the Wigner matrices for the other three rotations and we obtain

$$
\frac{1}{\left|G^{\prime}\right|} \sum_{R^{\prime}} D_{m^{\prime} m}^{\ell}\left(R^{\prime}\right)=\frac{1}{6} \sum_{j=1}^{6} D_{m^{\prime} m}^{\ell}\left(R_{j}\right)=\frac{\delta_{m^{\prime} m}+(-1)^{\ell-m} \delta_{m,-m^{\prime}}}{2},
$$

if $m$ and $m^{\prime}$ are integer multiples of 3, and zero otherwise. Equation (16) is then applied to a general fourth-rank tensor $T_{m}^{4}(3)$, where the argument (3) denotes the trigonal axes 
and we obtain the non-zero components of the symmetrized tensor $\left\langle T_{m}^{4}(3)\right\rangle$

$$
\begin{aligned}
& \left\langle T_{0}^{4}(3)\right\rangle=T_{0}^{4}(3), \\
& \left\langle T_{3}^{4}(3)\right\rangle=-\left\langle T_{-3}^{4}(3)\right\rangle=\frac{T_{3}^{4}(3)-T_{-3}^{4}(3)}{2} .
\end{aligned}
$$

For the second-rank tensor, all symmetrized components are zero, except for $\left\langle T_{0}^{2}(3)\right\rangle=$ $T_{0}^{2}(3)$. Of course, we have also the relation $\left\langle T_{0}^{0}(3)\right\rangle=T_{0}^{0}(3)$, which is valid for any group. Now we show that time-reversal symmetry implies that the symmetrized tensors are real. According to equation (3) $\left(T_{0}^{\ell}\right)^{*}=T_{0}^{\ell}$, so that $T_{0}^{\ell}$ is real. Still by equation (3) we have $\left(T_{3}^{\ell}\right)^{*}=-T_{-3}^{\ell}$. Thus, $\left\langle T_{3}^{4}(3)\right\rangle^{*}=\left(-T_{-3}^{4}(3)+T_{3}^{4}(3)\right) / 2=\left\langle T_{3}^{4}(3)\right\rangle$ is real as well.

In x-ray absorption spectra, the symmetrized tensors are spectral functions depending on the photon energy. For the example of the electric quadrupole transitions we take, for each energy $\hbar \omega$,

$$
T^{\ell}=\pi^{2} \alpha_{0} \sum_{f}\left\{\left\langle f\left|\{\mathbf{r} \otimes \mathbf{r}\}^{2}\right| i\right\rangle^{*} \otimes\left\langle f\left|\{\mathbf{r} \otimes \mathbf{r}\}^{2}\right| i\right\rangle\right\}^{\ell} \delta\left(E_{f}-E_{i}-\hbar \omega\right),
$$

where $\alpha_{0}$ is the fine structure constant, $E_{i}$ and $E_{f}$ the energy of the initial and final states. The symmetrized tensors $\left\langle T^{\ell}(3)\right\rangle$ can be calculated by multiplet programs. The value of these tensors for a chromium atom substituted for aluminum in spinel is given in figure 1] (see reference [1] for the details of the calculation)

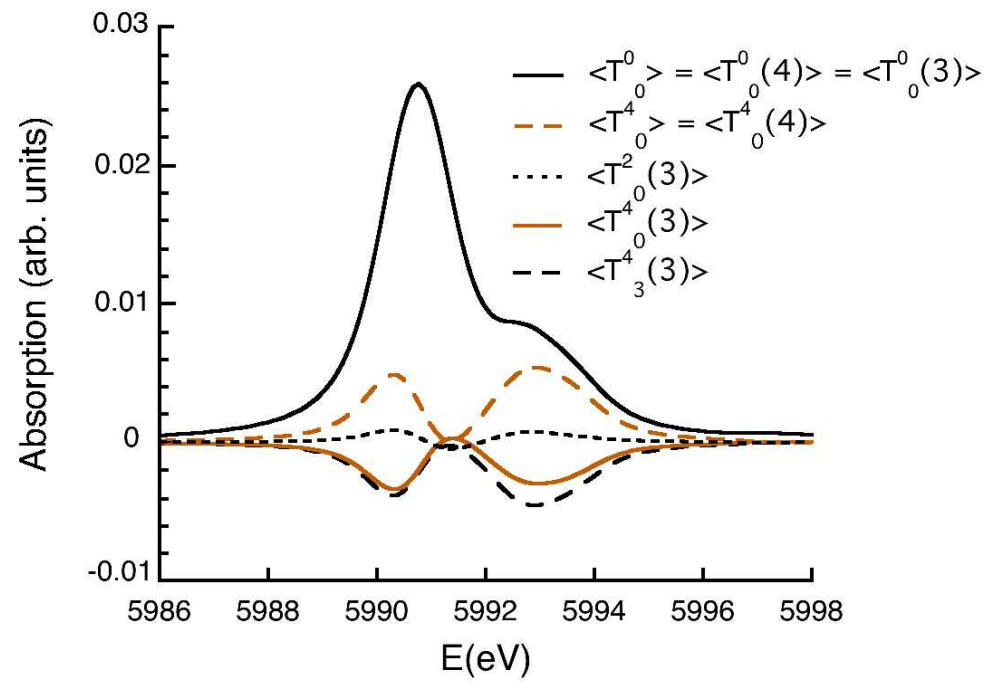

Figure 1. The symmetrized tensors $\left\langle T_{0}^{0}(3)\right\rangle,\left\langle T_{0}^{2}(3)\right\rangle,\left\langle T_{0}^{4}(3)\right\rangle$ and $\left\langle T_{3}^{4}(3)\right\rangle$ in the site frame, for the electric quadrupole transitions a the K-edge of a chromium atom substituted for aluminium in spinel. After averaging over the sites, only the symmetrized tensors $\left\langle T_{0}^{0}\right\rangle$ and $\left\langle T_{0}^{4}\right\rangle$ remain as independent parameters.

5.3.2. The crystal frame We consider now the same average in the crystal axes. The chosen $\mathrm{Al}$ site with reduced coordinates $(0,1 / 4,3 / 4)$ has a three-fold axis along the ($1,1,1)$ direction and a two-fold axis along the $(0,1,0)$ direction. Therefore, the six pure 
rotations of $D_{3 d}$ are now (i) the identity, denoted by $(x, y, z)$, (ii) a $C_{3}$ rotation about $(-1,1,1)$, denoted by $(-y, z,-x)$, (iii) its square $(-z,-x, y)$, (iv) a rotation of $\pi$ about $(1,1,0)$ denoted by $(y, x,-z),(\mathrm{v})$ a rotation of $\pi$ about $(1,0,1)$ denoted by $(z,-y, x)$, (vi) a rotation of $\pi$ about $(0,1,-1)$ denoted by $(-x,-z,-y)$. The notation used for the rotations is the result of the operation $R \mathbf{r}$ in the cubic axes. For example, the $C_{3}$ rotation gives

$$
R \mathbf{r}=\left(\begin{array}{ccc}
0 & -1 & 0 \\
0 & 0 & 1 \\
-1 & 0 & 0
\end{array}\right)\left(\begin{array}{l}
x \\
y \\
z
\end{array}\right)=\left(\begin{array}{c}
-y \\
z \\
-x
\end{array}\right) .
$$

The corresponding Euler angles are $(0,0,0),(\pi / 2, \pi / 2,0),(\pi, \pi / 2, \pi / 2),(0, \pi, \pi / 2)$, $(0, \pi / 2, \pi)$ and $(3 \pi / 2, \pi / 2,3 \pi / 2)$.

We apply again equation (16) to the general second-rank tensor $T_{m}^{2}(4)$, where the argument (4) stands for the cubic axes. This gives us the symmetrized tensor $\left\langle T^{2}(4)\right\rangle$

$$
\begin{aligned}
& \left\langle T_{0}^{2}(4)\right\rangle=0, \\
& \left\langle T_{-2}^{2}(4)\right\rangle=\left\langle T_{2}^{2}(4)\right\rangle^{*}=-i \lambda, \\
& \left\langle T_{-1}^{2}(4)\right\rangle=-\left\langle T_{1}^{2}(4)\right\rangle^{*}=(1+i) \lambda,
\end{aligned}
$$

with

$$
\lambda=\frac{\Im T_{2}^{2}(4)-\Re T_{1}^{2}(4)+\Im T_{1}^{2}(4)}{3},
$$

where we have used time-reversal symmetry as in equation (3). Note that $\lambda$ is real. For the tensor $\left\langle T^{4}(4)\right\rangle$,

$$
\begin{aligned}
\left\langle T_{0}^{4}(4)\right\rangle & =\sqrt{\frac{14}{5}}\left\langle T_{4}^{4}(4)\right\rangle=\sqrt{\frac{14}{5}}\left\langle T_{-4}^{4}(4)\right\rangle^{*}=\xi, \\
\left\langle T_{-3}^{4}(4)\right\rangle & =-\left\langle T_{3}^{4}(4)\right\rangle^{*}=(1-i) \sqrt{7} \zeta, \\
\left\langle T_{-2}^{4}(4)\right\rangle & =\left\langle T_{2}^{4}(4)\right\rangle^{*}=2 i \sqrt{2} \zeta \\
\left\langle T_{-1}^{4}(4)\right\rangle & =-\left\langle T_{1}^{4}(4)\right\rangle^{*}=(1+i) \zeta,
\end{aligned}
$$

with

$$
\begin{aligned}
& \xi=\frac{7 T_{0}^{4}(4)+\sqrt{70} \Re T_{4}^{4}(4)}{12}, \\
& \zeta=-\frac{\sqrt{7}\left(\Re T_{3}^{4}(4)+\Im T_{3}^{4}(4)\right)+2 \sqrt{2} \Im T_{2}^{4}(4)+\Re T_{1}^{4}(4)-\Im T_{1}^{4}(4)}{24} .
\end{aligned}
$$

Note that $\xi$ and $\zeta$ are real.

5.3.3. From site to crystal frame From this example, it is clear that the symmetrized tensor $\left\langle T^{\ell}(3)\right\rangle$ in the site frame is much simpler the same tensor $\left\langle T^{\ell}(4)\right\rangle$ in the crystal frame. The relation between the trigonal and cubic axes in Butler's tables is worked out in section 8.4 of the Appendix. 
Site symmetry and crystal symmetry

To go from one to the other we apply equation (17) and we obtain the relations

$$
\begin{aligned}
& \left\langle T_{0}^{4}(4)\right\rangle=-\frac{7\left\langle T_{0}^{4}(3)\right\rangle+2 \sqrt{70}\left\langle T_{3}^{4}(3)\right\rangle}{18}, \\
& \left\langle T_{-2}^{4}(4)\right\rangle=i \frac{\sqrt{10}\left\langle T_{0}^{4}(3)\right\rangle-\sqrt{7}\left\langle T_{3}^{4}(3)\right\rangle}{9} .
\end{aligned}
$$

We recover the relations (19) with

$$
\begin{aligned}
& \zeta=\frac{2 \sqrt{5}\left\langle T_{0}^{4}(3)\right\rangle-\sqrt{14}\left\langle T_{3}^{4}(3)\right\rangle}{36}, \\
& \xi=-\frac{7\left\langle T_{0}^{4}(3)\right\rangle+2 \sqrt{70}\left\langle T_{3}^{4}(3)\right\rangle}{18} .
\end{aligned}
$$

For the second-rank tensor we find the relations (18) with $\lambda=-\left\langle T_{0}^{2}(3)\right\rangle / \sqrt{6}$. For the zeroth-rank tensor we have obviously $\left\langle T_{0}^{0}(4)\right\rangle=\left\langle T_{0}^{0}(3)\right\rangle$.

\subsection{The example of garnet}

We consider now the $\mathrm{Al}$ site in garnet with the example of grossular $\mathrm{Ca}_{3} \mathrm{Al}_{2}\left(\mathrm{SiO}_{3}\right)_{4}$, which is a cubic mineral, with the space group $I a \overline{3} d$. The cubic cell contains 96 oxygen, 24 calcium, 24 silicium and 16 aluminium atoms. The $\mathrm{Al}^{3+}$ cations are at the $16 \mathrm{a}$ Wyckoff positions $(0,0,0)$. This site is a slightly distorted octahedral, with a small elongation along the (111) axis of the cube, and has the $\hat{3}\left(C_{3 i}\right)$ point symmetry.

We calculate the symmetrized tensor in the site frame as for spinel, but with the smaller symmetry group $C_{3 i}$.

We find that the non-zero tensor components are [10] $\left\langle T_{-3}^{4}(3)\right\rangle,\left\langle T_{0}^{4}(3)\right\rangle,\left\langle T_{3}^{4}(3)\right\rangle$, $\left\langle T_{0}^{2}(3)\right\rangle$ and $\left\langle T_{0}^{0}(3)\right\rangle$, as illustrated in figure 2 (see reference [17] for the details of the calculation).

It would not be very illuminating to calculate directly the symmetrized tensor in the crystal frame. It is more interesting to rotate the tensor. Indeed, in the group $C_{3 i}$, the $z$-axis is specified by the rotation axis, but the $y$-axis is arbitrary in the plane perpendicular to the rotation axis. This arbitrariness can be quite useful. For instance, the parametrization of the Hamiltonian is simplified by choosing the $y$ axis so that a crystal field parameter is set to zero (see Ref. [12] p. 184). This simplifies the calculation of the eigenstates but the parameter reappears as the angle $\alpha$ between the $y$ axis and the $(1,1,0)$ direction of the cube in the plane perpendicular to the $(-1,1,1)$ direction.

The corresponding rotation matrix is

$$
R=\sqrt{\frac{2}{3}}\left(\begin{array}{ccc}
\cos (\alpha+\pi / 3) & \cos (\alpha+2 \pi / 3) & \cos \alpha \\
\sin (\alpha+\pi / 3) & \sin (\alpha+2 \pi / 3) & \sin \alpha \\
-1 / \sqrt{2} & 1 / \sqrt{2} & 1 / \sqrt{2}
\end{array}\right) .
$$

The Euler angles are $\alpha, \arccos (1 / \sqrt{3}), \pi / 4$. The angle $\alpha$ describes a rotation about the axis $(-1,1,1)$. Therefore, the $\alpha$ dependency of the result is very simple: we have $T_{m}^{\ell}(\alpha)=T_{m}^{\ell}(0) \mathrm{e}^{-m i \alpha}$ because the corresponding Wigner matrix is $D_{m^{\prime} m}^{\ell}=\delta_{m m^{\prime}} \mathrm{e}^{-i m \alpha}$. 


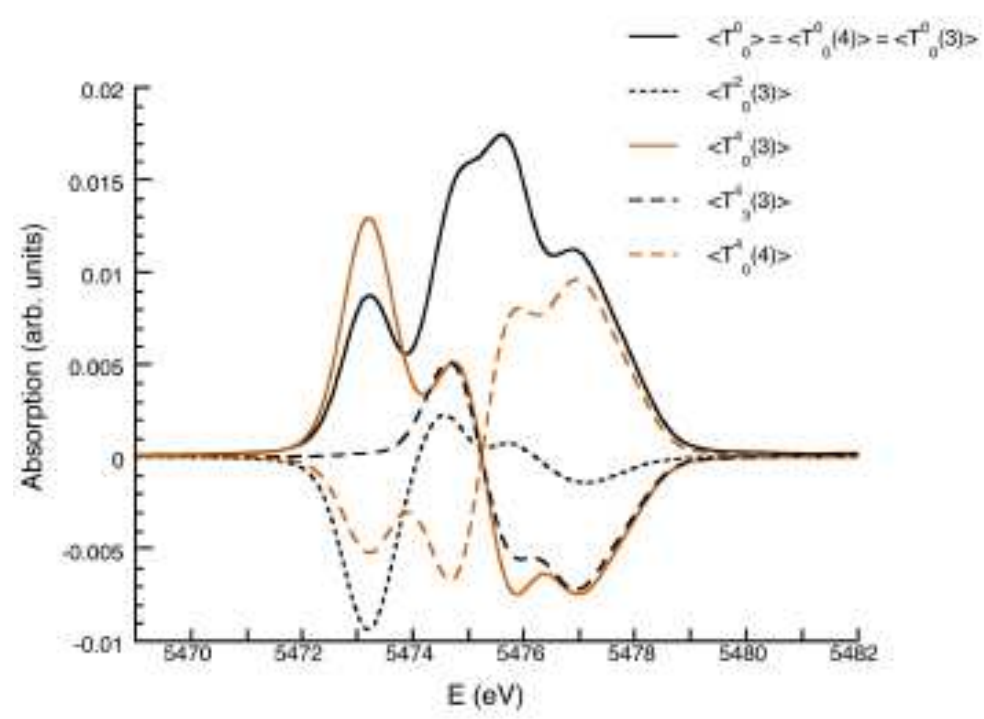

Figure 2. The symmetrized tensors $\left\langle T_{0}^{0}(3)\right\rangle,\left\langle T_{0}^{2}(3)\right\rangle,\left\langle T_{0}^{4}(3)\right\rangle$ and $\left\langle T_{3}^{4}(3)\right\rangle$ in the site frame, for the electric quadrupole transitions at the K-edge of a vanadium atom substituted for aluminium in grossular garnet.

To calculate the symmetrized tensor $\left\langle T_{m}^{4}(4)\right\rangle$ in the cubic axes, we use equation (17), we put $s=\left\langle T_{0}^{4}(3)\right\rangle$ and $t_{r}+i t_{i}=\mathrm{e}^{-i \alpha}\left\langle T_{3}^{4}(3)\right\rangle$ and we obtain

$$
\begin{aligned}
\left\langle T_{-4}^{4}(4)\right\rangle & =\left\langle T_{4}^{4}(4)\right\rangle^{*}=-\frac{\sqrt{70} s+20 t_{r}-12 i \sqrt{3} t_{i}}{36}, \\
\left\langle T_{-3}^{4}(4)\right\rangle & =-\left\langle T_{3}^{4}(4)\right\rangle^{*}=(1-i) \frac{2 \sqrt{35} s-7 \sqrt{2} t_{r}+3 i \sqrt{6} t_{i}}{36}, \\
\left\langle T_{-2}^{4}(4)\right\rangle & =\left\langle T_{2}^{4}(4)\right\rangle^{*}=i \frac{\sqrt{10} s-\sqrt{7} t_{r}}{9}, \\
\left\langle T_{-1}^{4}(4)\right\rangle & =-\left\langle T_{1}^{4}(4)\right\rangle^{*}=(1+i) \frac{2 \sqrt{5} s-\sqrt{14} t_{r}+3 i \sqrt{42} t_{i}}{36}, \\
\left\langle T_{0}^{4}(4)\right\rangle & =-\frac{7 s+2 \sqrt{70} t_{r}}{18} .
\end{aligned}
$$

The symmetrized second-rank tensor in the cubic frame is the same as for spinel.

The effect of the angle $\alpha$ on the experimental spectrum can be considerable, as is illustrated in figure 3 .

\section{From site symmetry to crystal symmetry}

We consider in this section another type of problem. We assume that we have calculated a symmetrized tensor $\left\langle T^{\ell}\right\rangle$ for a certain site $A$. We want to know the value of the same tensor for all the sites equivalent to $A$. In the first section, we describe how this can be done. In an x-ray absorption measurement, we measure the sum of the signals coming from all sites of the crystal. Therefore, we do not really need to know the spectrum of each site, we need to know the spectrum averaged over all the sites. Two methods will 
Site symmetry and crystal symmetry

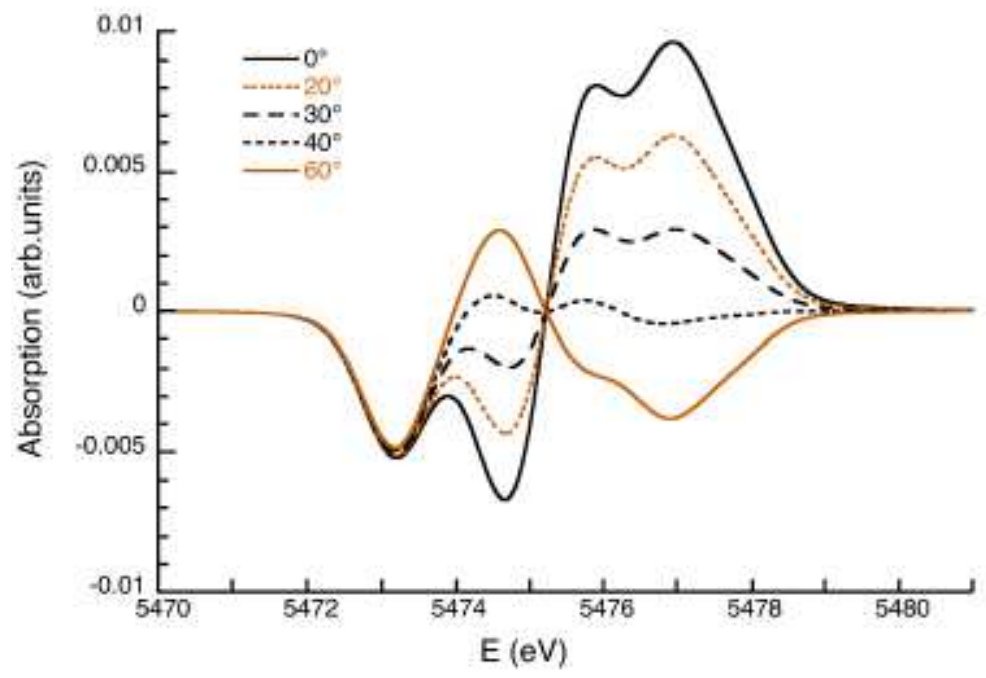

Figure 3. The crystal symmetrized tensor $\left\langle T_{0}^{4}\right\rangle$ as a function of $\alpha$, for the electric quadrupole transitions at the K-edge of a vanadium atom substituted for aluminium in grossular garnet.

be presented to do so: the coset method and the brute force method. Finally, we treat the examples of spinel and garnet.

\subsection{Changing site}

In general, the symmetries of the crystal make several equivalent sites. More precisely, if a crystal has the symmetry group $G$ and the site has symmetry group $G^{\prime}$, then the number of equivalent sites in the crystal is the ratio $|G| /\left|G^{\prime}\right|$. Assume that we have calculated a physical property described by a spherical tensor $T_{m}^{\ell}$ for a given site $A$. We want to calculate the properties of the equivalent site $B$.

If $\mathbf{r}_{A}$ and $\mathbf{r}_{B}$ are the position vectors of site $A$ and $B$, there is a symmetry operation $R$ of $G$ such that $\mathbf{r}_{B}=R \mathbf{r}_{A}$. If we denote by $G_{A}$ and $G_{B}$ the symmetry group of sites $A$ and $B$, then the symmetry operation $R^{\prime}$ of $G_{A}$ will be transformed into the operation $R R^{\prime} R^{-1}$ of $G_{B}$. This can be checked because $R$ transforms the rotation axis $\mathbf{n}$ of an operation $R^{\prime}$ of $G_{A}$ into the axis $R \mathbf{n}$, and $R R^{\prime} R^{-1}$ describes the rotation through the same angle as $R^{\prime}$ but about the axis $R \mathbf{n}$. This is indeed a symmetry operation of site $B$. Moreover, the tensor $T_{m}^{\ell}(B)$ at site $B$ is related to the tensor $T_{m}^{\ell}(A)$ at site $A$ by the relation

$$
T_{m}^{\ell}(B)=\sum_{m^{\prime}=-\ell}^{\ell} T_{m^{\prime}}^{\ell}(A) D_{m^{\prime} m}^{\ell}\left(R^{-1}\right) .
$$

We are now facing a typical subtelty of crystal symmetries. We could have expected the argument of the Wigner matrix to be $R$ instead of $R^{-1}$, but this is not the case because the rotation $R$ is in fact a passive operation from the point of view of site $B$. The rotation transports the rotation axis, which is a vector of the reference frame. By 
moving the atoms of the crystal, the rotation transports the reference frame of the site. Therefore, we are in the passive point of view and we need to use $R^{-1}$ because our convention uses the active point of view.

To check this, calculate the symmetrized tensor around $B$.

$$
\left\langle T_{m}^{\ell}(B)\right\rangle=\frac{1}{\left|G_{B}\right|} \sum_{R_{B}^{\prime} \in G_{B}} \sum_{m^{\prime}=-\ell}^{\ell} T_{m^{\prime}}^{\ell}(B) D_{m^{\prime} m}^{\ell}\left(R_{B}^{\prime}\right) .
$$

Now, we can use the fact that the operations $R_{B}^{\prime}$ of $G_{B}$ can be obtained from the operations $R_{A}^{\prime}$ of $G_{A}$ by $R_{B}^{\prime}=R R_{A}^{\prime} R^{-1}$. Therefore

$$
\left\langle T_{m}^{\ell}(B)\right\rangle=\frac{1}{\left|G_{A}\right|} \sum_{R_{A}^{\prime} \in G_{A}} \sum_{m^{\prime}=-\ell}^{\ell} T_{m^{\prime}}^{\ell}(B) D_{m^{\prime} m}^{\ell}\left(R R_{A}^{\prime} R^{-1}\right),
$$

where we used $\left|G_{A}\right|=\left|G_{B}\right|$, a consequence of the isomorphism between $G_{A}$ and $G_{B}$. We see that this is only compatible with the transformations $T^{\ell}(B)=T^{\ell}(A) D^{\ell}\left(R^{-1}\right)$ and $\left\langle T^{\ell}(B)\right\rangle=\left\langle T^{\ell}(A)\right\rangle D^{\ell}\left(R^{-1}\right)$.

\subsection{Changing the $x$-ray beam}

If we have calculated the spectrum of site $A$, it is possible to obtain the spectrum of any site equivalent to $A$ by calculating the spectrum of site $A$ for a rotated x-ray beam. This is physically clear because, if you rotate both the crystal and the x-ray, the spectrum does not change. Thus, if site $A$ is measured with a polarization $\epsilon$ and a wavevector $\mathbf{k}$, the spectrum of the site obtained by rotating $A$ with rotation matrix $R$ is the same as the spectrum of $A$ measured with a polarization $R^{-1} \boldsymbol{\epsilon}$ and a wavevector $R^{-1} \mathbf{k}$. To be fully convinced, we prove that, if $P^{a}$ and $Q^{a}$ are two ath-rank tensors, then $P^{a} \cdot\left(R Q^{a}\right)=\left(R^{-1} P^{a}\right) \cdot Q^{a}$. Equations (2) and (12) give us

$$
P^{a} \cdot\left(R Q^{a}\right)=P^{a} \cdot\left(Q^{a} D^{a}(R)\right)=\sum_{\alpha \beta}(-1)^{\alpha} P_{-\alpha}^{a} Q_{\beta}^{a} D_{\beta \alpha}^{a}(R) .
$$

The symmetry relation (see ref. [3], p. 79) $D_{\beta \alpha}^{a}(R)=(-1)^{\beta-\alpha} D_{-\alpha-\beta}^{a}\left(R^{-1}\right)$ enables us to write

$$
\begin{aligned}
P^{a} \cdot\left(R Q^{a}\right) & =\sum_{\alpha \beta}(-1)^{\beta} P_{-\alpha}^{a} D_{-\alpha-\beta}^{a}\left(R^{-1}\right) Q_{\beta}^{a} \\
& =\sum_{\beta}(-1)^{\beta}\left(P^{a} D^{a}\left(R^{-1}\right)\right)_{-\beta} Q_{\beta}^{a}=\left(R^{-1} P^{a}\right) \cdot Q^{a} .
\end{aligned}
$$

For the example of the electric dipole transition probability (14) we find

$$
\left\{\boldsymbol{\epsilon}^{*} \otimes \boldsymbol{\epsilon}\right\}^{a} \cdot R\left(\left\{\mathbf{r}_{f i}^{*} \otimes \mathbf{r}_{f i}\right\}^{a}\right)=R^{-1}\left(\left\{\boldsymbol{\epsilon}^{*} \otimes \boldsymbol{\epsilon}\right\}^{a}\right) \cdot\left\{\mathbf{r}_{f i}^{*} \otimes \mathbf{r}_{f i}\right\}^{a} .
$$

It remains to prove that the rotation of $\left\{\boldsymbol{\epsilon}^{*} \otimes \boldsymbol{\epsilon}\right\}^{a}$ corresponds to the rotation of $\boldsymbol{\epsilon}^{*}$ and

$\boldsymbol{\epsilon}$. This is done by using the following identity:

$$
R\left\{P^{a} \otimes Q^{b}\right\}^{c}=\left\{\left(R P^{a}\right) \otimes\left(R P^{b}\right)\right\}^{c} .
$$


To demonstrate the latter identity, we write the rotation in terms of the Wigner matrices and the coupled tensor in terms of the Clebsch-Gordan coefficients:

$$
R\left\{P^{a} \otimes Q^{b}\right\}_{\gamma}^{c}=\sum_{\alpha \beta \gamma^{\prime}}\left(a \alpha b \beta \mid c \gamma^{\prime}\right) P_{\alpha}^{a} Q_{\beta}^{b} D_{\gamma^{\prime} \gamma}^{c}(R) .
$$

The classical identity (30) transforms this expression into

$$
\begin{aligned}
R\left\{P^{a} \otimes Q^{b}\right\}_{\gamma}^{c} & =\sum_{\alpha \beta \alpha^{\prime} \beta^{\prime}}\left(a \alpha^{\prime} b \beta^{\prime} \mid c \gamma\right) P_{\alpha}^{a} Q_{\beta}^{b} D_{\alpha \alpha^{\prime}}^{a}(R) D_{\beta \beta^{\prime}}^{b}(R) \\
& =\sum_{\alpha^{\prime} \beta^{\prime}}\left(a \alpha^{\prime} b \beta^{\prime} \mid c \gamma\right)(R P)_{\alpha^{\prime}}^{a}(R Q)_{\beta^{\prime}}^{b}=\left\{\left(R P^{a}\right) \otimes\left(R P^{b}\right)\right\}_{\gamma}^{c} .
\end{aligned}
$$

Equations (23) and (24) yield

$$
\left\{\boldsymbol{\epsilon}^{*} \otimes \boldsymbol{\epsilon}\right\}^{a} \cdot R\left(\left\{\mathbf{r}_{f i}^{*} \otimes \mathbf{r}_{f i}\right\}^{a}\right)=\left\{\left(R^{-1} \boldsymbol{\epsilon}^{*}\right) \otimes\left(R^{-1} \boldsymbol{\epsilon}\right)\right\}^{a} \cdot\left\{\mathbf{r}_{f i}^{*} \otimes \mathbf{r}_{f i}\right\}^{a} .
$$

In other words, rotating the crystal by $R$ gives the same result as rotating the x-ray beam by $R^{-1}$. The same result is true for the electric quadrupole transition probabilities, except for the fact that the polarization $\mathbf{k}$ are rotated by $R^{-1}$ and not only $\boldsymbol{\epsilon}$.

\subsection{The coset method}

The coset method is a powerful way to calculate the the tensor symmetrized over the crystal from the tensor symmetrized over a single site. We first introduce some mathematical concepts.

6.3.1. Mathematical aspects The symmetry group $G_{A}$ of a site $A$ is a subgroup of the symmetry group $G$ of the crystal. The number of sites equivalent to $A$ in the unit cell is the ratio $|G| /\left|G_{A}\right|$, where $|G|$ and $\left|G_{A}\right|$ denote the number of elements of $G$ and $G_{A}$. This ratio, denoted by $n$ in the following, is an integer by the Euler-Lagrange theorem ([7] p. 11). We can pick up $n$ symmetry operations $g_{i}$ in $G$ that transport site $A$ to the $n$ sites equivalent to $A$.

We first introduce some mathematical concepts [7]. If $g$ is an element of a group $G$ and $G_{A}$ a subgroup of $G$, the set $g G_{A}=\left\{g h: h \in G_{A}\right\}$ is called a coset. If we take two elements $g$ and $g^{\prime}$ of $G$, then either $g G_{A}$ and $g^{\prime} G_{A}$ are either identical or disjoint (i.e. they have no element in common). The number of different cosets is $n=|G| /\left|G_{A}\right|$ and the number of elements in each coset is $\left|G_{A}\right|$. Moreover, every element of $G$ belongs to one and only one coset. Therefore, if we pick up any element $g_{i}$ in each coset, we have $G=g_{1} G_{A} \cup \ldots \cup g_{n} G_{A}$ and each $g_{i}$ is called a representative of its coset.

6.3.2. Cosets in a crystal We apply these concepts to a crystal. The crystal symmetry group is a space group $G$. The symmetry group of a site $A$ is the set of operations of $G$ that leave site $A$ invariant. More precisely, if $\mathbf{r}_{A}$ is the coordinate vector of site $A$, then $G_{A}=\left\{g \in G \mid g\left(\mathbf{r}_{A}\right)=\mathbf{r}_{A}\right\}$. It is clear that $G_{A}$ is a subgroup of $G$. If $n=|G| /\left|G_{A}\right|$, there are $n$ sites in the crystal that are equivalent to $A$. The group $G$ is partitioned 
into $n$ cosets $g_{1} G_{A}, \ldots, g_{n} G_{A}$. All the elements of a given coset send site $A$ to the same equivalent site. Take an element $g_{i}$ in each coset $g_{i} G_{A}$.

The symmetrized tensor $\left\langle T^{\ell}\right\rangle_{X}$ over the full crystal is obtained from the sitesymmetrized tensor $\left\langle T^{\ell}\right\rangle_{A}$ by the operation

$$
\left\langle T^{\ell}\right\rangle_{X}=\frac{1}{n} \sum_{i=1}^{n}\left\langle T^{\ell}\right\rangle_{A} D^{\ell}\left(g_{i}^{-1}\right)
$$

where, for a space group operation $g, D^{\ell}(g)$ is the Wigner matrix corresponding to the proper rotation of $g$. Equation (25) can be described by saying that we average over the symmetries of site $A$, then we go to another sites with $g_{i}$ and we average over the symmetries of that other site.

We show now that equation (25) gives the same result as an average over all crystal symmety operations. We already know that

$$
\left\langle T^{\ell}\right\rangle_{A}=\frac{1}{\left|G_{A}\right|} \sum_{h \in G_{A}} T^{\ell} D^{\ell}(h)=\frac{1}{\left|G_{A}\right|} \sum_{h \in G_{A}} T^{\ell} D^{\ell}\left(h^{-1}\right),
$$

because the sum over the elements of a group is the same as the sum over the inverse elements of this group. Therefore

$$
\begin{aligned}
\left\langle T^{\ell}\right\rangle_{X} & =\frac{1}{n\left|G_{A}\right|} \sum_{i=1}^{n} \sum_{h \in G_{A}} T^{\ell} D^{\ell}\left(h^{-1}\right) D^{\ell}\left(g_{i}^{-1}\right)=\frac{1}{|G|} \sum_{i=1}^{n} \sum_{h \in G_{A}} T^{\ell} D^{\ell}\left(h^{-1} g_{i}^{-1}\right) \\
& =\frac{1}{|G|} \sum_{i=1}^{n} \sum_{h \in G_{A}} T^{\ell} D^{\ell}\left(\left(g_{i} h\right)^{-1}\right)=\frac{1}{|G|} \sum_{g \in G} T^{\ell} D^{\ell}(g),
\end{aligned}
$$

where we used $\left(g_{i} h\right)^{-1}=h^{-1} g_{i}^{-1}$ and $|G|=n\left|G_{A}\right|$. Note that the proof holds because we used sets $g_{i} G_{A}$ (i.e. left cosets) and not $G_{A} g_{i}$ (i.e. right cosets).

In other words, the average over the site symmetries followed by the average over the sites gives the average over the crystal symmetries. This can be considered as a factorization of the average, because $\sum_{g} D^{\ell}(g)=\sum_{h} D^{\ell}(h) \sum_{i} D^{\ell}\left(g_{i}^{-1}\right)$. It can be checked easily that the result of equation (25) is the same if we replace $g_{i}$ by any $g_{i}^{\prime}$ in $g_{i} G_{A}$. We illustrate this by our two favorite examples, spinel and garnet.

6.3.3. The example of spinel We call site $A$ the site $(0,1 / 4,3 / 4)$ of the spinel structure. The space group $G$ has 192 operation $\&$. Twelve of them leave site A invariant: $(x, y, z),(-y+3 / 4, z,-x+3 / 4),(-z+3 / 4,-x+3 / 4, y),(y+1 / 4, x+3 / 4,-z+1 / 2)$, $(z+1 / 4,-y+1 / 2, x+3 / 4),(-x,-z+1 / 2,-y+1 / 2),(-x,-y+1 / 2,-z+1 / 2)$, $(y+1 / 4,-z+1 / 2, x+3 / 4),(z+1 / 4, x+3 / 4,-y+1 / 2),(-y+3 / 4,-x+3 / 4, z)$, $(-z+3 / 4, y,-x+3 / 4)$, and $(x, z, y)$. This set of twelve operations is a group isomorphic

$\S$ The full space group has of course an infinite number of operations because it contains all the translations by a vector of the Bravais lattice. We consider here the group $G$ obtained as the quotient of the full space group by the group of translations of the simple cubic lattice. We could have used the quotient of the full space group by the group of translations of the fcc lattice (which has only 48 operations), however our choice is simpler from the programming point of view: we take the symmetry operations given by the table [16, and we identify two points whose coordinates differ by an integer. 
to $D_{3 d}$. The isomorphism $\varphi$ is described explicitly as follows. If $\mathbf{r}_{A}$ is the coordinate vector of site $A$, for any operation $g$ of the set, we define the operation $\varphi(g)$ by $\varphi(g)(\mathbf{r})=g\left(\mathbf{r}+\mathbf{r}_{A}\right)-\mathbf{r}_{A}$. It is an isomorphism because $\varphi\left(g^{\prime} g\right)=\varphi\left(g^{\prime}\right) \varphi(g)$ :

$$
\varphi\left(g^{\prime}\right) \varphi(g)(\mathbf{r})=\varphi\left(g^{\prime}\right)\left(g\left(\mathbf{r}+\mathbf{r}_{A}\right)-\mathbf{r}_{A}\right)=\left(g^{\prime} g\right)\left(\mathbf{r}+\mathbf{r}_{A}\right)-\mathbf{r}_{A}=\varphi\left(g^{\prime} g\right)(\mathbf{r}),
$$

and it can be checked that the image by $\varphi$ of the first six operations are the rotations listed in section 5.3.2, the image of the other six operations are the same rotations multiplied by -1 . The space group operations $g$ are selected by the condition that the site is fixed: $g\left(\mathbf{r}_{A}\right)=\mathbf{r}_{A}$. Thus, the origin is a fixed point of $\varphi(g): \varphi(g)(0)=g\left(\mathbf{r}_{A}\right)-\mathbf{r}_{A}=0$.

There are 16 sites equivalent to $A$ because the ratio $|F d \overline{3} m| /\left|D_{3 d}\right|$ is 16 . However, each site is equivalent to three other sites by pure lattice translations $(1 / 2,1 / 2,0)$, $(1 / 2,0,1 / 2)$ and $(0,1 / 2,1 / 2)$. The x-ray spectrum of these sites will be equal because their orientations with respect to the x-ray beam are the same. Therefore, we are left with 4 equivalent sites: $A$ itself and the sites with coordinates $(1 / 4,3 / 4,0),(3 / 4,0,1 / 4)$ and $(1 / 2,1 / 2,1 / 2)$. A representative of the coset corresponding to each of these sites is $(-y+1 / 2, x+3 / 4, z+1 / 4),(-x+3 / 4,-y+3 / 4, z)$ and $(y+1 / 4,-x, z+1 / 4)$.

6.3.4. The example of garnet We consider now site $A$ as the $A l$ site in grossular garnet with the three-fold axis along the $(-1,1,1)$ direction. Its coordinates are $(1 / 2,1 / 2,0)$. It is left invariant by the following six operations: identity: $(x, y, z)$, rotation through $2 \pi / 3$ about the $(-1,1,1)$ axis: $(-y, z+1 / 2,-x+1 / 2)$, rotation through $4 \pi / 3$ about the $(-1,1,1)$ axis: $(-z+1 / 2,-x, y+1 / 2)$, and the same operations multiplied by an inversion $(-x,-y,-z),(y,-z+1 / 2, x+1 / 2)$ and $(z+1 / 2, x,-y+1 / 2)$. This group is $C_{3 i}$. The number of cosets (i.e. the number of sites equivalent to $A$ ) is $|\operatorname{Ia} \overline{3} d| /\left|C_{3 i}\right|=16$. If we remove the lattice translation $(1 / 2,1 / 2,1 / 2)$ we are left with 8 equivalent sites.

The approach in terms of cosets is quite powerful in practice because it completely avoids the explicit description of the sites and of the symmetry operations that transform a site into another one. Many programs compute the symmetry operations of the sites (for example PWSCF). It is then enough to take one of these sites, to choose any representative $g_{i}$ in each coset and to calculate the contribution of all equivalent sites by the formula $\left\langle T^{\ell}\right\rangle_{A} D^{\ell}\left(g_{i}^{-1}\right)$. The average over the crystal is then obtained with equation (25).

\subsection{The brute force method}

If one is not interested in the contribution of each site to the spectrum of the crystal, a still simpler solution is to take the average of $\left\langle T^{\ell}\right\rangle_{A}$ over all the symmetry operations of the crystal. This is not very clever because the site operations have already been taken into account and we average over them a second time, but this method is well suited for computers.

We show now that averaging the site-symmetrized tensor over all the symmetry 
operations of the crystal gives the same result as the coset method.

$$
\begin{aligned}
\frac{1}{|G|} \sum_{g \in G}\left\langle T^{\ell}\right\rangle_{A} D^{\ell}(g) & =\frac{1}{\left|G_{A}\right||G|} \sum_{h \in G_{A}} \sum_{g \in G} T^{\ell} D^{\ell}(h) D^{\ell}(g) \\
& =\frac{1}{n\left|G_{A}\right|^{2}} \sum_{h, h^{\prime} \in G_{A}} \sum_{i} T^{\ell} D^{\ell}(h) D^{\ell}\left(h^{\prime}\right) D^{\ell}\left(g_{i}^{-1}\right) \\
& =\frac{1}{n\left|G_{A}\right|} \sum_{h \in G_{A}} \sum_{i} T^{\ell} D^{\ell}(h) D^{\ell}\left(g_{i}^{-1}\right)=\left\langle T^{\ell}\right\rangle_{X},
\end{aligned}
$$

where we used the identity $\sum_{h, h^{\prime} \in G_{A}} D^{\ell}\left(h h^{\prime}\right)=\left|G_{A}\right| \sum_{h \in G_{A}} D^{\ell}(h)$, which is readily established. Therefore, the average over all the symmetry operations of the crystal gives the same result as the average over the sites, irrespective of the number of equivalent sites.

\subsection{The case of spinel}

We illustrate the coset method with the case of spinel. We first notice that the absorption cross section is invariant under a translation of the Bravais lattice, because such a translation multiplies the wavefunction by a phase (independent of $\mathbf{r}$ ) that disappears in the square modulus. Therefore, by removing the translations, we can replace the representatives of the four cosets given in section 6.3 .3 by the four rotations about the $z$-axis of the crystal through angles $0, \pi / 2, \pi$ and $3 \pi / 2$. For a fourth-rank tensor, the average over the coset operations is rather drastic. The only non-zero elements of the matrix $M=(1 / 4) \sum_{i} D^{4}\left(g_{i}^{-1}\right)$ are $M(-4,-4)=M(0,0)=M(4,4)=1$. Therefore, the crystal-symmetrized fourth-rank tensor is $\left\langle T^{4}\right\rangle_{X}=\left\langle T^{4}(4)\right\rangle M$ :

$$
\left\langle T_{0}^{4}\right\rangle_{X}=\sqrt{\frac{14}{5}}\left\langle T_{4}^{4}\right\rangle_{X}=\sqrt{\frac{14}{5}}\left\langle T_{-4}^{4}\right\rangle_{X}=\left\langle T_{0}^{4}(4)\right\rangle .
$$

The relation between $\left\langle T_{0}^{4}(4)\right\rangle$ and the site-symmetrized tensor in the trigonal axes is given by equation (20).

For a second-rank tensor, the matrix $M=(1 / 4) \sum_{i} D^{2}\left(g_{i}^{-1}\right)$ has a single non-zero element: $M(0,0)=1$. Therefore $\left\langle T_{0}^{2}\right\rangle_{X}=0$, as expected [10].

\subsection{The case of garnet}

We illustrate the brute force method with the case of garnet. To calculate the spherical tensor of garnet, we use the brute force method and calculate $M=(1 / 48) \sum_{R} D^{\ell}(R)$, where the sum runs over all the symmetry operations of the cube.

For $\ell=4$, the only nonzero matrix elements are

$$
\begin{aligned}
& M(-4,-4)=M(-4,4)=M(4,-4)=M(4,4)=\frac{5}{24}, \\
& M(-4,0)=M(0,-4)=M(0,4)=M(4,0)=\frac{\sqrt{70}}{24}, \\
& M(0,0)=\frac{7}{12} .
\end{aligned}
$$


Therefore, the only non-zero components of a fourth-rank tensor are

$$
\left\langle T_{0}^{4}\right\rangle_{X}=\sqrt{\frac{14}{5}}\left\langle T_{4}^{4}\right\rangle_{X}=\sqrt{\frac{14}{5}}\left\langle T_{-4}^{4}\right\rangle_{X}=\left\langle T_{0}^{4}(4)\right\rangle=-\frac{7 s+2 \sqrt{70} t_{r}}{18},
$$

where we recall that $s=\left\langle T_{0}^{4}(3)\right\rangle$ and $t_{r}=(1 / 2)\left(\mathrm{e}^{-3 i \alpha}\left\langle T_{3}^{4}(3)\right\rangle-\mathrm{e}^{3 i \alpha}\left\langle T_{-3}^{4}(3)\right\rangle\right)$. Note that $s$ and $t_{r}$ are real because of time-reversal symmetry.

\section{Spherical average}

In Cartesian coordinates, the electric quadrupole matrix elements can be written, for linearly polarized x-rays

$$
\left|T_{f i}\right|^{2}=\sum_{i j l m} \boldsymbol{\epsilon}_{i} k_{j} \boldsymbol{\epsilon}_{l} k_{m} \sigma_{i j l m}
$$

with $\sigma_{i j l m}=\left\langle i\left|x_{i} x_{j}\right| f\right\rangle\left\langle f\left|x_{l} x_{m}\right| i\right\rangle$. For a powder sample we have the spherical average

$$
\begin{aligned}
\left\langle\left|T_{f i}\right|^{2}\right\rangle= & \frac{1}{30}\left(2 \sigma_{x x x x}+2 \sigma_{y y y y}+2 \sigma_{z z z z}+6 \sigma_{x y x y}+6 \sigma_{x z x z}+6 \sigma_{y z y z}\right. \\
& \left.-\sigma_{x x y y}-\sigma_{x x z z}-\sigma_{y y x x}-\sigma_{z z x x}-\sigma_{y y z z}-\sigma_{z z y y}\right) .
\end{aligned}
$$

If the system is non magnetic, then $\sigma_{i i j j}=\sigma_{j j i i}$ and the average further simplifies

$$
\begin{aligned}
\left\langle\left|T_{f i}\right|^{2}\right\rangle= & \frac{1}{15}\left(\sigma_{x x x x}+\sigma_{y y y y}+\sigma_{z z z z}+3 \sigma_{x y x y}+3 \sigma_{x z x z}+3 \sigma_{y z y z}\right. \\
& \left.-\sigma_{x x y y}-\sigma_{x x z z}-\sigma_{y y z z}\right) .
\end{aligned}
$$

\section{Formulas}

\subsection{Rotation matrix}

The rotation of $\psi$ around the direction $\mathbf{n}$ (a unit vector) is represented by the rotation matrix (see Ref.[2], p. 10) $R=\operatorname{Id}+\sin \psi N+(1-\cos \psi) N^{2}$, where $N$ is the skewsymmetric matrix with matrix elements $N_{i j}=-\sum_{k} \epsilon_{i j k} n_{k}$, so that $\left(N^{2}\right)_{i j}=n_{i} n_{j}-\delta_{i j}$.

Conversely, the rotation angle $\psi$ and the rotation axis $\mathbf{n}$ are determined from the rotation matrix $R$ by the relations $\cos \psi=(\operatorname{tr} R-1) / 2, n_{1} \sin \psi=\left(R_{32}-R_{23}\right) / 2$, $n_{2} \sin \psi=\left(R_{13}-R_{31}\right) / 2$, and $n_{3} \sin \psi=\left(R_{21}-R_{12}\right) / 2$. This is a corrected version of the relation given in Ref. [2] p. 20.

\subsubsection{Euler angles Ref. [2], p. 24}

$$
R=\left(\begin{array}{ccc}
c_{\alpha} c_{\beta} c_{\gamma}-s_{\alpha} s_{\gamma} & -c_{\alpha} c_{\beta} s_{\gamma}-s_{\alpha} c_{\gamma} & c_{\alpha} s_{\beta} \\
s_{\alpha} c_{\beta} c_{\gamma}+c_{\alpha} s_{\gamma} & -s_{\alpha} c_{\beta} s_{\gamma}+c_{\alpha} c_{\gamma} & s_{\alpha} s_{\beta} \\
-s_{\beta} c_{\gamma} & s_{\beta} s_{\gamma} & c_{\beta}
\end{array}\right),
$$

where $0 \leq \alpha<2 \pi, 0 \leq \beta \leq \pi$ and $0 \leq \gamma<2 \pi, c_{\alpha}=\cos \alpha, s_{\alpha}=\sin \alpha$, etc. There is a one-to-one correspondence between rotations and parameters in this range, except for the case $\beta=0$ and $\beta=\pi$, which describe the rotation through the angle $\alpha+\gamma$ and $\alpha-\gamma$, respectively, about the axis $(0,0,1)$. 
8.1.2. Euler-Rodrigues parameters Although the Euler angles are more common, the Euler-Rodrigues have the advantage of being expressed in terms of the rotation matrix without using circular functions. Thus, it can be convenient to derive analytical expressions. From the axis $\mathbf{n}$ and the angle $\psi$, we define the Euler-Rodrigues parameters (Ref. 2] p. 54). $\alpha_{0}=\cos (\psi / 2), \alpha_{i}=\sin (\psi / 2) n_{i}$. These parameters are interesting for analytic calculations because they can be derived from the rotation matrix without using trigonometric functions ([2] p. 19): If $\operatorname{tr} R \neq-1$, then $\alpha_{0}=\sqrt{\operatorname{tr} R+1} / 2$, $\alpha_{1}=\left(R_{32}-R_{23}\right) /\left(4 \alpha_{0}\right), \alpha_{2}=\left(R_{13}-R_{31}\right) /\left(4 \alpha_{0}\right)$, and $\alpha_{3}=\left(R_{21}-R_{12}\right) /\left(4 \alpha_{0}\right)$. If $\operatorname{tr} R=-1$, then $\alpha_{0}=0$ and $\alpha_{i}=\left(\operatorname{sign} \alpha_{i}\right) \sqrt{\left(1+R_{i i}\right) / 2}$ for $i=1,2,3$ with $\operatorname{sign} \alpha_{1}=1$, $\operatorname{sign} \alpha_{2}=\operatorname{sign} R_{12}, \operatorname{sign} \alpha_{3}=\operatorname{sign} R_{13}$.

$$
R=\left(\begin{array}{ccc}
\alpha_{0}^{2}+\alpha_{1}^{2}-\alpha_{2}^{2}-\alpha_{3}^{2} & 2 \alpha_{1} \alpha_{2}-2 \alpha_{0} \alpha_{3} & 2 \alpha_{1} \alpha_{3}+2 \alpha_{0} \alpha_{2} \\
2 \alpha_{1} \alpha_{2}+2 \alpha_{0} \alpha_{3} & \alpha_{0}^{2}+\alpha_{2}^{2}-\alpha_{3}^{2}-\alpha_{1}^{2} & 2 \alpha_{2} \alpha_{3}-2 \alpha_{0} \alpha_{1} \\
2 \alpha_{1} \alpha_{3}-2 \alpha_{0} \alpha_{2} & 2 \alpha_{2} \alpha_{3}+2 \alpha_{0} \alpha_{1} & \alpha_{0}^{2}+\alpha_{3}^{2}-\alpha_{1}^{2}-\alpha_{2}^{2}
\end{array}\right)
$$

where $\alpha_{0}=\cos (\psi / 2), \alpha_{i}=\sin (\psi / 2) n_{i}$.

\subsection{Solid harmonics}

For a vector $\mathbf{r}=(x, y, z)$, the solid harmonics $Y_{\ell}^{m}(\mathbf{r})$ are defined by (see Ref. [2] p. 71)

$$
Y_{\ell}^{m}(\mathbf{r})=\sqrt{\frac{(2 \ell+1)(\ell+m) !(\ell-m) !}{4 \pi}} \sum_{k} \frac{(-x-i y)^{k+m}(x+i y)^{k} z^{\ell-2 k-m}}{2^{2 k+m}(k+m) ! k !(l-m-2 k) !},
$$

where $k$ runs from $\max (0,-m)$ to the integer part of $(\ell-m) / 2$. The most important example of solid harmonics is

$$
Y_{1}(\mathbf{r})=\sqrt{\frac{3}{4 \pi}}\left(\begin{array}{c}
\frac{x-i y}{\sqrt{2}} \\
z \\
-\frac{x+i y}{\sqrt{2}}
\end{array}\right)
$$

where the upper component is $Y_{1}^{-1}(\mathbf{r})$.

\subsection{Wigner matrices}

There are several representations of the Wigner rotation matrices. We present here the expressions in terms of Euler angles and of Euler-Rodrigues parameters. Other formulas have been derived, for example the recent invariant spinor representation [18].

8.3.1. Euler angles For a rotation $R$ expressed in terms of Euler angles $\alpha, \beta$, $\gamma$, the Wigner matrix is ([2] p. 46)

$$
D_{m^{\prime} m}^{\ell}(R)=\mathrm{e}^{-i m^{\prime} \alpha} d_{m^{\prime} m}^{\ell}(\beta) \mathrm{e}^{-i m \gamma} .
$$

Various expressions exist for the reduced Wigner matrix $d_{m^{\prime} m}^{\ell}(\beta)$. The following formula (valid for half-integer $\ell$ ) is particularly convenient for computers, because it avoids the 
presence of singular terms ([2] p. 50):

$$
d_{m^{\prime} m}^{\ell}(\beta)=(-1)^{\lambda} \sqrt{\frac{k !(2 \ell-k) !}{(k+\mu) !(k+\nu) !}}\left(\sin \frac{\beta}{2}\right)^{\mu}\left(\cos \frac{\beta}{2}\right)^{\nu} P_{k}^{(\mu, \nu)}(\cos \beta),
$$

where $k=\min \left(\ell+m, \ell-m, \ell+m^{\prime}, \ell-m^{\prime}\right)$ and the non-negative integers $\mu, \nu$ and $\lambda$ are determined by the value of $k$

- if $k=\ell+m$, then $\mu=m^{\prime}-m, \nu=-m^{\prime}-m$ and $\lambda=m^{\prime}-m^{\prime}$,

- if $k=\ell-m$, then $\mu=m-m^{\prime}, \nu=m^{\prime}+m$ and $\lambda=0$,

- if $k=\ell+m^{\prime}$, then $\mu=m-m^{\prime}, \nu=-m^{\prime}-m$ and $\lambda=0$,

- if $k=\ell-m^{\prime}$, then $\mu=m^{\prime}-m, \nu=-m^{\prime}+m$ and $\lambda=m^{\prime}-m^{\prime}$.

In this expression, the only possible numerical difficulty occurs for $0^{0}$ that should be 1 . The Jacobi polynomials $P_{k}^{(\mu, \nu)}(x)$ are given by the formula

$$
P_{k}^{(\mu, \nu)}(x)=\sum_{i=0}^{k}\left(\begin{array}{c}
k+\mu \\
i
\end{array}\right)\left(\begin{array}{c}
k+\nu \\
k-i
\end{array}\right)\left(\frac{x-1}{2}\right)^{k-i}\left(\frac{x+1}{2}\right)^{i} .
$$

For example, the Wigner matrix for first-rank tensors is

$$
D^{1}=\left(\begin{array}{ccc}
\frac{\cos \beta+1}{2} \mathrm{e}^{i(\alpha+\gamma)} & \frac{\sin \beta}{\sqrt{2}} \mathrm{e}^{i \alpha} & \frac{\cos \beta-1}{2} \mathrm{e}^{i(\alpha-\gamma)} \\
-\frac{\sin \beta}{\sqrt{2}} \mathrm{e}^{i \gamma} & \cos \beta & \frac{\sin \beta}{\sqrt{2}} \mathrm{e}^{-i \gamma} \\
\frac{\cos \beta-1}{2} \mathrm{e}^{i(\gamma-\alpha)} & -\frac{\sin \beta}{\sqrt{2}} \mathrm{e}^{-i \alpha} & \frac{\cos \beta+1}{2} \mathrm{e}^{-i(\alpha+\gamma)}
\end{array}\right),
$$

where the upper left matrix element is $D_{-1-1}^{1}$. Two useful special cases are $d_{m m^{\prime}}^{\ell}(0)=$ $\delta_{m m^{\prime}}$ and $d_{m m^{\prime}}^{\ell}(\pi)=(-1)^{\ell+m} \delta_{m,-m^{\prime}}$.

\subsubsection{Euler-Rodrigues parameters The Wigner rotation matrix is}

$$
\begin{aligned}
& D_{m^{\prime} m}^{\ell}(R)=\sqrt{\left(\ell+m^{\prime}\right) !\left(\ell-m^{\prime}\right) !(\ell+m) !(\ell-m) !} \\
& \quad \sum_{k} \frac{\left(\alpha_{0}-i \alpha_{3}\right)^{\ell+m-k}\left(-i \alpha_{1}-\alpha_{2}\right)^{m^{\prime}-m+k}\left(-i \alpha_{1}+\alpha_{2}\right)^{k}\left(\alpha_{0}+i \alpha_{3}\right)^{\ell-m^{\prime}-k}}{(\ell+m-k) !\left(m^{\prime}-m+k\right) ! k !\left(\ell-m^{\prime}-k\right) !}
\end{aligned}
$$

where $k$ runs from $\max \left(0, m-m^{\prime}\right)$ to $\min \left(\ell+m, \ell-m^{\prime}\right)$.

\subsection{Butler's orientation}

The powerful multiplet program developed by Theo Thole and Barry Searle is based on Butler's conventions. For the calculation of trigonal sites in cubic crystals, it is necessary to know precisely the relation between the cubic and trigonal reference frames, which is not clearly stated in Butler's book. To determine it, we combine Butler's tables pp. 522, 527 and 549 of [12]. This shows that the transition between spherical harmonics $|1 \mathrm{~m}\rangle_{3}$ in the trigonal axes (i.e. in the $O-D_{3}-C_{3}$ basis) and spherical harmonics $|1 m\rangle_{4}$ in the cubic axes (i.e. in the $O-D_{4}-C_{4}$ basis) is

$$
|1-1\rangle_{3}=|1-1\rangle_{4} \frac{(1-i)(\sqrt{3}+1)}{\sqrt{24}}+|10\rangle_{4} \frac{1}{\sqrt{3}}+|11\rangle_{4} \frac{(1+i)(\sqrt{3}-1)}{\sqrt{24}},
$$


Site symmetry and crystal symmetry

$$
\begin{aligned}
|10\rangle_{3} & =|1-1\rangle_{4} \frac{-1+i}{\sqrt{6}}+|10\rangle_{4} \frac{1}{\sqrt{3}}+|11\rangle_{4} \frac{1+i}{\sqrt{6}} \\
|11\rangle_{3} & =|1-1\rangle_{4} \frac{(1-i)(\sqrt{3}-1)}{\sqrt{24}}+|10\rangle_{4} \frac{-1}{\sqrt{3}}+|11\rangle_{4} \frac{(1+i)(\sqrt{3}+1)}{\sqrt{24}} .
\end{aligned}
$$

This can be rewritten

$$
|1 m\rangle_{3}=\sum_{m^{\prime}=-1}^{1}\left|1 m^{\prime}\right\rangle_{4} D_{m^{\prime} m}^{1}(R),
$$

for the rotation $R$ corresponding to the Euler angles $\alpha=3 \pi / 4, \beta=\arccos (1 / \sqrt{3})$ and $\gamma=\pi$. This corresponds to the $C_{3 z}$ axis of $D_{3}$ along the $(-1,1,1)$ direction of the cube and the $C_{2 y}$ axis of $D_{3}$ along the $(1,1,0)$ direction of the cube (see fig. 11.6 of Ref. [12], p. 204). The inverse rotation has Euler angles $\left(0, \beta_{0}, \pi / 4\right)$. To be still more detailed, the rotation

$$
R=R\left(3 \pi / 4, \beta_{0}, \pi\right)=\left(\begin{array}{ccc}
1 / \sqrt{6} & 1 / \sqrt{2} & -1 / \sqrt{3} \\
-1 / \sqrt{6} & 1 / \sqrt{2} & 1 / \sqrt{3} \\
\sqrt{2 / 3} & 0 & 1 / \sqrt{3}
\end{array}\right),
$$

transforms any symmetry operation $R^{\prime}$ in the $D_{3 d}$ axes into the symmetry operation $R R^{\prime} R^{-1}$ in the cubic axes.

\section{Coupling identities}

We gather some useful coupling formulas. If $\mathbf{a}, \mathbf{b}, \mathbf{c}$ and $\mathbf{d}$ are vectors, we denote by $\mathbf{a}^{1}, \mathbf{b}^{1}, \mathbf{c}^{1}$ and $\mathbf{d}^{1}$ the corresponding first-rank spherical tensors. Then, according to reference [3] p. 66 and 67 ,

$$
\begin{aligned}
\left\{\mathbf{a}^{1} \otimes \mathbf{b}^{1}\right\}^{1}=\frac{i}{\sqrt{2}}(\mathbf{a} \times \mathbf{b})^{1} . & \\
\left\{\left\{\mathbf{a}^{1} \otimes \mathbf{b}^{1}\right\}^{0} \otimes\left\{\mathbf{c}^{1} \otimes \mathbf{d}^{1}\right\}^{0}\right\}^{0}= & \frac{1}{3}(\mathbf{a} \cdot \mathbf{b})(\mathbf{c} \cdot \mathbf{d}) . \\
\left\{\left\{\mathbf{a}^{1} \otimes \mathbf{b}^{1}\right\}^{2} \otimes\left\{\mathbf{c}^{1} \otimes \mathbf{d}^{1}\right\}^{2}\right\}^{0}= & \frac{1}{\sqrt{5}}\left(\frac{1}{2}(\mathbf{a} \cdot \mathbf{c})(\mathbf{b} \cdot \mathbf{d})+\frac{1}{2}(\mathbf{a} \cdot \mathbf{d})(\mathbf{b} \cdot \mathbf{c})\right. \\
& \left.-\frac{1}{2}(\mathbf{a} \cdot \mathbf{b})(\mathbf{c} \cdot \mathbf{d})\right) .
\end{aligned}
$$

To prove equation (13), we start from the identity

$$
\begin{aligned}
\left\{P^{a} \otimes Q^{b}\right\}^{c} \cdot\left\{R^{d} \otimes S^{e}\right\}^{c}= & (-1)^{2 a+b-d} \sum_{g}(2 c+1)\left\{\begin{array}{lll}
a & b & c \\
e & d & g
\end{array}\right\} \\
& \times\left\{P^{a} \otimes R^{d}\right\}^{g} \cdot\left\{Q^{b} \otimes S^{e}\right\}^{g},
\end{aligned}
$$

where $g$ runs between $\max (|a-d|,|b-e|)$ and $\min (a+d, b+e)$ (see equation (13) p. 70 of [3]). Equation (13) corresponds to the case $c=0$ because of the special value of the $6 j$-symbol (equation (1) p. 299 of [3])

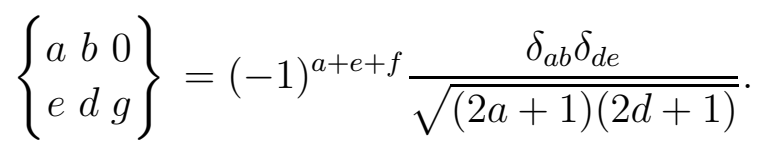


The interplay between Wigner matrices and Clebsch-Gordan coefficients is described by the following identity (equation (5) p. 85 of [3])

$$
\sum_{\gamma^{\prime}}\left(a \alpha b \beta \mid c \gamma^{\prime}\right) D_{\gamma^{\prime} \gamma}^{c}(R)=\sum_{\alpha^{\prime} \beta^{\prime}}\left(a \alpha^{\prime} b \beta^{\prime} \mid c \gamma\right) D_{\alpha \alpha^{\prime}}^{a}(R) D_{\beta \beta^{\prime}}^{c}(R) .
$$

\section{Acknowledgments}

We thank Etienne Balan and Philippe Sainctavit for very constructive comments.

\section{References}

[1] Juhin A, Brouder C, Arrio M A, Cabaret D, Sainctavit P, Balan E, Bordage A, Calas G, Eeckhout S G and Glatzel P 2008 X-ray natural dichroism in cubic compounds: the case of substitutional $\mathrm{Cr}^{3+}$ in $\mathrm{MgAl}_{2} \mathrm{O}_{4}$ To be published

[2] Biedenharn L and Louck J 1981 Angular Momentum in Quantum Physics vol. 8 of Encyclopedia of Mathematics and its Applications (Reading: Addison-Wesley)

[3] Varshalovich D A, Moskalev A N and Khersonskii V K 1988 Quantum Theory of Angular Momentum (Singapore: World Scientific)

[4] Goulon J 1989 Systèmes moléculaires : Dichroïsme circulaire naturel et magnétique dans les spectroscopies optiques ou d'excitation des couches internes in E Beaurepaire, B Carrière and J P Kappler, eds., Rayonnement synchrotron polarisé, électrons polarisés et magnétisme (Strasbourg: IPCMS) pp. 333-86

[5] Natoli C, Brouder C, Sainctavit P, Goulon J, Goulon-Ginet C and Rogalev A 1998 Calculation of x-ray natural circular dichroism Europ. Phys. J. B 4 1-11

[6] Carra P and Benoist R R 2000 X-ray natural circular dichroism Phys. Rev. B 62 R7703-6

[7] Ludwig W and Falter C 1996 Symmetries in Physics: Group Theory applied to Physical Problems 2nd ed. (Berlin: Springer)

[8] Carra P, Jerez A and Marri I 2003 X-ray dichroism in noncentrosymmetric crystals Phys. Rev. B 67045111

[9] Marri I and Carra P 2004 Scattering operators for E1-E2 x-ray resonant diffraction Phys. Rev. B 69113101

[10] Brouder C 1990 Angular dependence of x-ray absorption spectra J. Phys.: Condens. Matter 2 701-38

[11] Cowan R D 1981 The Theory of Atomic Structure and Spectra (Berkeley: University of Calivornia Press)

[12] Butler P H 1981 Point Symmetry Group Applications (New York: Plenum Press)

[13] Thole B T, van der Laan G, Fuggle J C, Sawatzky G A, Karnatak R C and Esteva J M $10853 d$ x-ray-absorption lines and the $3 d^{9} 4 f^{n+1}$ multiplets of lanthanides Phys. Rev. B 32 5107-18

[14] Kotani A, Ogasawara H, Okada K, Thole B T and Sawatzky G A 1989 Theory of multiplet structure in 4d core photoabsorption spectra of $\mathrm{CeO}_{2}$ Phys. Rev. B 40 65-73

[15] Kuiper P, Searle B G, Rudolf P, Tjeng L H and Chen C T 1957 X-ray magnetic dichroism and antiferromagnetic $\mathrm{Fe}_{2} \mathrm{O}_{3}$ : The orientation of magnetic moments observed by Fe $2 p$ x-rayabsorption spectroscopy Phys. Rev. Lett. 70 1549-52

[16] Hahn T 2002 International Tables for Crystallography: Volume A 5th ed. (Dordrecht: Kluwer Academic Publishers)

[17] Bordage A and coll 2008 Substitutional chromium in garnet In preparation

[18] Manakov N L, Meremianin A V and Starace A F 2001 Invariant spinor representations of finite rotation matrices Phys. Rev. A 64032105 